\title{
Some exact controllability results for the linear KdV equation and uniform controllability in the zero-dispersion limit
}

\author{
O. Glass* \& S. Guerrero*
}

February 20, 2008

\begin{abstract}
In this paper, we deal with controllability properties of linear and nonlinear Korteweg-de Vries equations in a bounded interval. The main part of this paper is a result of uniform controllability of a linear KdV equation in the limit of zero-dispersion. Moreover, we establish a result of null controllability for the linear equation via the left Dirichlet boundary condition, and of exact controllability via both Dirichlet boundary conditions. As a consequence, we obtain some local exact controllability results for the nonlinear KdV equation.
\end{abstract}

\section{Introduction}

In this paper, we are interested in two types of controllability results concerning a linearized Korteweg-de Vries equation. These two types are the following.

- Mainly, we are interested in how the cost of the null controllability of a linear Korteweg-de Vries $(\mathrm{KdV})$ evolves as the dispersive term is brought to $0^{+}$(Theorem 1$)$. In the case of the vanishing viscosity limit (that is, when a dissipative term is considered rather than a dispersive one), this problem has been studied in [7] and [11].

- Next, we consider the problem of exact controllability for this equation, when the dispersion coefficient is fixed (Theorems 2 and 3). Such results yield results of local exact controllability for the usual (nonlinear) KdV equation (Theorems 4 and 5). The controllability of the KdV equation has already been studied in several papers, see in particular $[15,16,17,18,19]$.

Let us be more specific on the problem under view. Let $T>0$ be a given final time. Our system is the following one:

$$
\begin{cases}y_{t}+\nu y_{x x x}+(M y)_{x}=0 & \text { in }(0, T) \times(0,1), \\ y_{\mid x=0}=v_{1}, y_{\mid x=1}=v_{2}, y_{x \mid x=1}=v_{3} & \text { in }(0, T), \\ y_{\mid t=0}=y_{0} & \text { in }(0,1) .\end{cases}
$$

Here, $\nu$ is a positive dispersion coefficient, $M=M(t, x)$ is a transport coefficient (constant in the main problem), $v_{i}(i=1,2,3)$ are time-dependent functions which constitute the controls of our system. Observe that the classical $\mathrm{KdV}$ equation corresponds to $M(t, x)=1+\frac{y}{2}(t, x)$.

The principal result which we consider in this paper is the problem of uniform controllability of equation (1) (where $M$ is a constant) as the dispersion parameter tends to $0^{+}$. Of course, one can hope to reach such a property only when the limit system (obtained by setting $\nu=0$ in (1)) is controllable. In this situation, this means $M \neq 0$ and the time of controllability $T$ is greater than $1 /|M|$. Due to the effect of the dispersive term (which is strongly asymmetric), we are able to obtain a result only in the case $M<0$. Moreover, we consider a time of controllability which is of the form $K_{0} /|M|$, but our proof does not apply for any $K_{0}>1$ (such a limitation appears also in the case of vanishing viscosity, see $[7,11])$. Our result is the following.

\footnotetext{
${ }^{*}$ Université Pierre et Marie Curie-Paris6, UMR 7598 Laboratoire Jacques-Louis Lions, Paris, F-75005 France. E-mails: glass@ann.jussieu.fr, guerrero@ann.jussieu.fr.
} 
Theorem 1. There exists a positive constant $K_{0}$ such that for any negative constant $M$, there exists $\nu_{0}>0$ such that for any $T \geq K_{0} /|M|$, any $y_{0} \in W^{1, \infty}(0,1)$ and any $\nu \in\left(0, \nu_{0}\right)$, there exist $v_{1}^{\nu}, v_{2}^{\nu}, v_{3}^{\nu} \in$ $L^{2}(0, T)$ such that the solution $y \in L^{2}((0, T) \times(0,1)) \cap C^{0}\left([0, T] ; H^{-1}(0,1)\right)$ of $(1)$ satisfies $y_{\mid t=T}=0$ in $(0,1)$ and moreover the controls are uniform in $\nu$ in the sense that

$$
\left\|v_{1}^{\nu}\right\|_{L^{2}(0, T)}+\left\|v_{2}^{\nu}\right\|_{L^{2}(0, T)}+\left\|v_{3}^{\nu}\right\|_{L^{2}(0, T)} \leq K_{1}\left\|y_{0}\right\|_{W^{1, \infty}(0,1)}
$$

for a constant $K_{1}>0$ independent of $\nu$ and $y_{0}$.

Remark 1. As far as we know, the question of uniform (local exact) controllability of the KdV equation (5) in the limit $\nu \rightarrow 0^{+}$, is an open problem. In the case of a vanishing viscosity limit for Burgers equation, such a result was established in [10].

Next, we consider the problem of controllability of (1) for fixed $\nu$. We obtain the following two results in that case. The first one (Theorem 2 below) is a result of zero-controllability for equation (1) with $M$ constant. Equivalently, this establishes the controllability on the trajectories of equation (1). This is done by using only the Dirichlet condition on the left of the domain (the other conditions are kept null). A similar result has been obtained by L. Rosier [17].

Our second result (Theorem 3 below) is an exact controllability result for equation (1) with $M$ constant. Here this is done by using two controls, namely both Dirichlet conditions on the left and on the right of the domain (the Neumann condition on the right is kept null).

Theorem 2. Let $M$ be a constant and $\nu>0$ be fixed. Then, for any $y_{0} \in H^{-1}(0,1)$, there exists $v_{1} \in L^{2}(0, T)$ such that the solution $y \in L^{2}((0, T) \times(0,1)) \cap C^{0}\left([0, T] ; H^{-1}(0,1)\right)$ of $(1)$ with $v_{2}=v_{3}=0$ satisfies $y_{\mid t=T}=0$ in $(0,1)$. Moreover, for any $\nu \in(0,1)$, there exists $C^{*}>0$ such that

$$
\left\|v_{1}\right\|_{L^{2}(0, T)}^{2} \leq \frac{C^{*}}{\nu}\left\|y_{0}\right\|_{H^{-1}(0,1)}^{2}
$$

Besides, for $\nu$ suitably small (in terms of $M$ only), one can estimate $C^{*}$ as follows:

$$
C^{*}=\exp \left\{\frac{\widetilde{C}|M|^{1 / 2}}{\nu^{1 / 2}}\left(1+\frac{1}{T^{1 / 2}|M|^{1 / 2}}\right)\right\},
$$

where $\widetilde{C}>0$ is a constant independent of $M, \nu$ and $y_{0}$.

Remark 2. Theorem 2 is essentially due to L. Rosier [17], but here we require less regularity on $y_{0}$ and give a different estimate on the size of the control. Precisely, our constant is of order $\exp \left(C /(\nu T)^{1 / 2}\right)$ instead of order $\exp (C /(\nu T))$, which is essential in the analysis of Theorem 1.

Theorem 3. Let $M$ be a constant and $\nu>0$ be fixed. Then, for any $y_{0}, y_{1} \in L^{2}(0,1)$, there exist $v_{1}$ and $v_{2}$ in $L^{2}(0, T)$ such that the solution $y \in L^{2}((0, T) \times(0,1)) \cap C^{0}\left([0, T] ; H^{-1}(0,1)\right)$ of $(1)$ with $v_{3}=0$ satisfies $y_{\mid t=T}=y_{1}$ in $(0,1)$.

Remark 3. These results are independent of the length of the interval. This is to be compared to [15], where L. Rosier considers the case $v_{1}=v_{2}=0$ (that is, a control acting via the right Neumann boundary condition). In that case, the equation for $M=1$ is controllable if and only if the length of the interval does not belong to a countable critical set.

Remark 4. These results could also be established when $M$ is a function depending on $(t, x)$, belonging to $L^{2}\left(0, T ; H^{1}(0,1)\right) \cap C^{0}\left([0, T] ; L^{2}(0,1)\right)$, or even $L^{2}\left(0, T ; L^{\infty}(0,1)\right) \cap L^{\infty}\left(0, T ; L^{2}(0,1)\right)$ (see Paragraph 2.2.2, Equations (50)-(51) and Proposition 5).

As a natural consequence of an exact controllability result for the linearized system, one can usually prove a local exact controllability result for the nonlinear system. Here, as corollaries of Theorems 2 and 3, we get the following results for the Korteweg-de Vries equation. The first one (Theorem 4) is a result of local exact controllability on trajectories where the control acts upon the left Dirichlet boundary condition, while the second one (Theorem 5) is a result of local exact controllability via both Dirichlet conditions. 
Theorem 4. Let $\nu>0$ be fixed. For $\bar{y}_{0} \in L^{2}(0,1)$, we consider $\bar{y} \in L^{\infty}\left(0, T ; L^{2}(0,1)\right) \cap L^{2}\left(0, T ; H_{0}^{1}(0,1)\right)$ the solution of

$$
\begin{cases}\bar{y}_{t}+\bar{y}_{x}+\overline{y y}_{x}+\nu \bar{y}_{x x x}=0 & \text { in }(0, T) \times(0,1), \\ \bar{y}_{\mid x=0}=0, \bar{y}_{\mid x=1}=0, \bar{y}_{x \mid x=1}=0 & \text { in }(0, T), \\ \bar{y}_{\mid t=0}=\bar{y}_{0} & \text { in }(0,1) .\end{cases}
$$

Then, there exists $\delta>0$ such that for any $y_{0} \in L^{2}(0,1)$ satisfying $\left\|y_{0}-\bar{y}_{0}\right\|_{L^{2}(0,1)} \leq \delta$, there exists $v_{1} \in H^{1 / 2-\varepsilon}(0, T)$ for any $\varepsilon>0$, such that the solution $y \in L^{2}\left(0, T ; H^{1}(0,1)\right) \cap C^{0}\left([0, T] ; L^{2}(0,1)\right)$ of

$$
\begin{cases}y_{t}+y_{x}+y y_{x}+\nu y_{x x x}=0 & \text { in }(0, T) \times(0,1), \\ y_{\mid x=0}=v_{1}, y_{\mid x=1}=0, y_{x \mid x=1}=0 & \text { in }(0, T), \\ y_{\mid t=0}=y_{0} & \text { in }(0,1),\end{cases}
$$

satisfies $y_{\mid t=T}=\bar{y}_{\mid t=T}$ in $(0,1)$.

Remark 5. The same result (with a slightly different functional framework) has been previously obtained by L. Rosier [17].

Theorem 5. Let $\nu>0$ be fixed. There exists $\mu>0$ such that for any $y_{0}, y_{1} \in L^{2}(0,1)$ satisfying

$$
\left\|y_{0}\right\|_{L^{2}(0,1)}+\left\|y_{1}\right\|_{L^{2}(0,1)}<\mu,
$$

there exists $v_{1}, v_{2} \in L^{2}(0, T)$ such that the solution $y \in L^{2}((0, T) \times(0,1)) \cap C^{0}\left([0, T] ; H^{-1}(0,1)\right)$ of

$$
\begin{cases}y_{t}+y_{x}+y y_{x}+\nu y_{x x x}=0 & \text { in }(0, T) \times(0,1), \\ y_{\mid x=0}=v_{1}, y_{\mid x=1}=v_{2}, y_{x \mid x=1}=0 & \text { in }(0, T), \\ y_{\mid t=0}=y_{0} & \text { in }(0,1),\end{cases}
$$

satisfies $y_{\mid t=T}=y_{1}$ in $(0,1)$.

Remark 6. Note that, despite Remark 3, the $K d V$ equation is locally controllable with $v_{1}=v_{2}=0$, even for critical lengths, see [6, 3, 4].

This paper is organized as follows. In Section 2, we study the Cauchy problem (1). In Section 3, we establish Theorems 2 and 3, via some observability inequalities (following the classical HUM method, see [14]). In Section 4 we establish the nonlinear results, Theorems 4 and 5 . Section 5 is devoted to the proof of Theorem 1. Finally, we have put the proofs of some technical properties in Section 6 .

\section{Cauchy problem}

In this section, we explain what we mean by a solution of (1) and we prove regularity results for such a solution.

\subsection{Statement of the results}

Definition 1. Given $T>0, y_{0} \in H^{-1}(0,1)$ and $\left(v_{1}, v_{2}, v_{3}\right) \in\left[L^{2}(0, T)\right]^{2} \times H^{-1 / 3}(0, T)$, we call y a solution (by transposition) of (1), a function $y \in L^{2}((0, T) \times(0,1))$ satisfying

$$
\begin{aligned}
\int_{0}^{T} \int_{0}^{1} y f d x d t=\left\langle y_{0},\left.u\right|_{t=0}\right\rangle_{H^{-1}(0,1) \times H_{0}^{1}(0,1)}+\nu \int_{0}^{T} v_{1} u_{x x \mid x=0} d t-\nu \int_{0}^{T} v_{2} u_{x x \mid x=1} d t \\
+\nu\left\langle v_{3}, u_{x \mid x=1}\right\rangle_{H^{-1 / 3}(0, T) \times H^{1 / 3}(0, T)}, \quad \forall f \in L^{2}((0, T) \times(0,1)),
\end{aligned}
$$

where $u$ is the solution of

$$
\begin{cases}-u_{t}-\nu u_{x x x}-M u_{x}=f & \text { in }(0, T) \times(0,1), \\ u_{\mid x=0}=u_{\mid x=1}=u_{x \mid x=0}=0 & \text { in }(0, T) \\ u_{\mid t=T}=0 & \text { in }(0,1) .\end{cases}
$$


Remark 7. We will see that the function $u$ in (9) belongs to $L^{2}\left(0, T ; H^{2}(0,1)\right)$. Moreover, the definiteness of the above terms in (8) (in particular the terms concerning $u_{x x \mid x=0}$ and $u_{x x \mid x=1}$, see (51)) will follow from regularity results established below.

Now our results concerning the existence, uniqueness and regularity of the solutions of the Cauchy problem for equation (1) according to this definition, are given in the following three propositions.

Proposition 1. Assume that $M$ is constant. Let $y_{0} \in H^{-1}(0,1), v_{1}, v_{2} \in L^{2}(0, T)$ and $v_{3} \in H^{-1 / 3}(0, T)$. Then there exists a unique solution $y \in L^{2}((0, T) \times(0,1))$ of $(1)$ such that

$$
\|y\|_{L^{2}((0, T) \times(0,1))} \leq(C / \nu)\left(\left\|y_{0}\right\|_{H^{-1}(0,1)}+\left\|v_{1}\right\|_{L^{2}(0, T)}+\left\|v_{2}\right\|_{L^{2}(0, T)}+\left\|v_{3}\right\|_{H^{-1 / 3}(0, T)}\right),
$$

for some constant $C>0$ independent of $y_{0}, v_{1}, v_{2}, v_{3}$ and $\nu$.

Proposition 2. Assume that $M$ is constant. Let $y_{0} \in H^{-1}(0,1), v_{1}, v_{2} \in L^{2}(0, T)$ and $v_{3} \in H^{-1 / 3}(0, T)$ as in Proposition 1. Then the solution $y \in L^{2}((0, T) \times(0,1))$ of $(1)$ belongs to $C\left([0, T] ; H^{-1}(0,1)\right)$, and moreover it satisfies the following estimate:

$$
\|y\|_{L^{\infty}\left(0, T ; H^{-1}(0,1)\right)} \leq(C / \nu)\left(\left\|y_{0}\right\|_{H^{-1}(0,1)}+\left\|v_{1}\right\|_{L^{2}(0, T)}+\left\|v_{2}\right\|_{L^{2}(0, T)}+\left\|v_{3}\right\|_{H^{-1 / 3}(0, T)}\right),
$$

for some constant $C>0$ independent of $y_{0}, v_{1}, v_{2}, v_{3}$ and $\nu$.

Remark 8. Observe that Proposition 2 does not follow straightforwardly from Proposition 1. In fact, from Proposition 1 and equation (1) we have that $y \in C\left([0, T] ; H^{-3 / 2}(0,1)\right)$ with a suitable estimate. But in order to prove the continuity in time with values in $H^{-1}(0,1)$, we need a further analysis.

Remark 9. Concerning the general inhomogeneous Cauchy boundary problem for KdV, let us cite [12] (see also [2, 5, 8]), where conditions

$$
v_{1}, v_{2} \in H^{(1+s) / 3}(0, T) \quad \text { and } \quad v_{3} \in H^{s / 3}(0, T), \quad s>-3 / 4,
$$

are required in order to define a solution.

Propositions 1 and 2 can be extended to the case where $M$ is variable as follows.

Proposition 3. Consider $M \in Y_{1 / 4}:=L^{2}\left(0, T ; H^{1}(0,1)\right) \cap C^{0}\left([0, T] ; L^{2}(0,1)\right)$. Let $y_{0} \in H^{-1}(0,1)$, $v_{1}, v_{2} \in L^{2}(0, T)$ and $v_{3} \in H^{-1 / 3}(0, T)$. Then there exists a unique solution $y \in Y_{0}:=L^{2}((0, T) \times$ $(0,1)) \cap C\left([0, T] ; H^{-1}(0,1)\right)$ of $(1)$ such that

$$
\|y\|_{Y_{0}} \leq C\left(\left\|y_{0}\right\|_{H^{-1}(0,1)}+\left\|v_{1}\right\|_{L^{2}(0, T)}+\left\|v_{2}\right\|_{L^{2}(0, T)}+\left\|v_{3}\right\|_{H^{-1 / 3}(0, T)}\right),
$$

for some constant $C>0$ depending on $\nu$ and $\|M\|_{Y_{1 / 4}}$ but independent of $y_{0}, v_{1}, v_{2}$ and $v_{3}$.

The notations $Y_{0}$ and $Y_{1 / 4}$ will be justified at the beginning of Paragraph 2.3.

Remark 10. Let us underline that in Proposition 3 we did not specify the dependence of $C$ with respect to $\nu$, since it is not necessary for our purpose. In the case of Propositions 1 and 2, this dependence is of polynomial type in $1 / \nu$; in the case of Proposition 3, looking at the proof more closely, one can see that this constant is (at most) of exponential type.

The proofs of Propositions 1, 2 and 3 are done simultaneously. They rely on estimates for the adjoint system (9), which are of two different types. The first one is a standard energy estimate. The second one is an improved regularity result for system (9) with $M=0$. Using these two estimates and interpolation arguments, we prove that, whether $f$ is taken in $L^{2}((0, T) \times(0,1))$ or in $L^{1}\left(0, T ; H_{0}^{1}(0,1)\right)$, the solution of (9) satisfies

$$
u_{\mid t=0} \in H_{0}^{1}(0,1), u_{x \mid x=1} \in H^{1 / 3}(0, T) \quad \text { and } \quad u_{x x \mid x=0}, u_{x x \mid x=1} \in L^{2}(0, T),
$$

with appropriate estimates.

We develop these two kinds of estimates in separate paragraphs. Finally in a last step we combine the two kinds of estimates to conclude. 


\section{$2.2 \quad$ Energy estimate}

In this paragraph we prove that for $f \in L^{2}\left(0, T ; H^{-1}(0,1)\right) \cup L^{1}\left(0, T ; L^{2}(0,1)\right)$, the solution of (9) belongs to the space

$$
Y_{1 / 4}:=L^{2}\left(0, T ; H^{1}(0,1)\right) \cap C^{0}\left([0, T] ; L^{2}(0,1)\right),
$$

together with some hidden regularity and suitable estimates. These estimates are slightly different in the case where $M$ is constant and in the case where $M$ depends on $(t, x)$.

\subsubsection{The case where $M$ is constant}

Here we prove that there exists a positive constant $C$ (independent of $\nu$ ) such that

$$
\|u\|_{L^{\infty}\left(0, T ; L^{2}(0,1)\right)}+\nu^{1 / 2}\|u\|_{L^{2}\left(0, T ; H^{1}(0,1)\right)}+\nu^{1 / 2}\left\|u_{x \mid x=1}\right\|_{L^{2}(0, T)} \leq\left(C / \nu^{1 / 2}\right)\|f\|_{L^{2}\left(0, T ; H^{-1}(0,1)\right)},
$$

and

$$
\|u\|_{L^{\infty}\left(0, T ; L^{2}(0,1)\right)}+\nu^{1 / 2}\|u\|_{L^{2}\left(0, T ; H^{1}(0,1)\right)}+\nu^{1 / 2}\left\|u_{x \mid x=1}\right\|_{L^{2}(0, T)} \leq C\|f\|_{L^{1}\left(0, T ; L^{2}(0,1)\right)} .
$$

In order to prove (12) and (13), we will suppose that $f$ belongs to $C_{0}^{\infty}((0, T) \times(0,1))$. By an argument of density, this immediately establishes (12) (resp. (13)) for general $f \in L^{2}\left(0, T ; H^{-1}(0,1)\right)$ (resp. for general $\left.f \in L^{1}\left(0, T ; L^{2}(0,1)\right)\right)$. Unless otherwise stated, we will denote by $C$ various positive constants which depend only on $M$ and $T$ (and in particular not on $\nu$ ).

- First case: $f \in L^{2}\left(0, T ; H^{-1}(0,1)\right)$. Here, we show estimate (12).

Let us multiply equation (9) by $(1-x) u$ and integrate in $x$. We get

$$
\begin{aligned}
-\frac{1}{2} \frac{d}{d t} \int_{0}^{1}(1-x)|u|^{2} d x+\nu \int_{0}^{1}\left((1-x) u_{x} u_{x x}-u u_{x x}\right) d x & -\frac{M}{2} \int_{0}^{1}|u|^{2} d x \\
& =\langle f,(1-x) u\rangle_{H^{-1}(0,1) \times H_{0}^{1}(0,1)} .
\end{aligned}
$$

We integrate by parts again:

$$
\begin{array}{r}
-\frac{1}{2} \frac{d}{d t} \int_{0}^{1}(1-x)|u|^{2} d x+\frac{3}{2} \nu \int_{0}^{1}\left|u_{x}\right|^{2} d x=\frac{M}{2} \int_{0}^{1}|u|^{2} d x+\langle f,(1-x) u\rangle_{H^{-1}(0,1) \times H_{0}^{1}(0,1)} \\
\leq C \int_{0}^{1}|u|^{2} d x+\frac{\nu}{2} \int_{0}^{1}\left|u_{x}\right|^{2} d x+(C / \nu)\|f(t)\|_{H^{-1}(0,1)}^{2} .
\end{array}
$$

The estimate in the $C^{0}\left([0, T] ; L^{2}(0,1)\right)$-norm is obtained by multiplying equation (9) by $u$ :

This yields:

$$
-\frac{1}{2} \frac{d}{d t} \int_{0}^{1}|u|^{2} d x+\nu \int_{0}^{1} u_{x} u_{x x} d x=\langle f, u\rangle_{H^{-1}(0,1) \times H_{0}^{1}(0,1)} .
$$

$$
-\frac{1}{2} \frac{d}{d t} \int_{0}^{1}|u|^{2} d x+\frac{\nu}{2}\left|u_{x \mid x=1}\right|^{2}=\langle f, u\rangle_{H^{-1}(0,1) \times H_{0}^{1}(0,1)} .
$$

Combining (15) and (16), we get the existence of a positive constant $C$ such that

$$
-\frac{1}{2} \frac{d}{d t}\left(e^{C t} \int_{0}^{1}(2-x)|u|^{2} d x\right)+\frac{\nu e^{C t}}{2} \int_{0}^{1}\left|u_{x}\right|^{2} d x+\frac{\nu e^{C t}}{2}\left|u_{x \mid x=1}\right|^{2} \leq\left(C e^{C t} / \nu\right)\|f(t)\|_{H^{-1}(0,1)}^{2} .
$$

Finally, integrating between $t$ and $T$, we obtain estimate (12).

- Second case: $f \in L^{1}\left(0, T ; L^{2}(0,1)\right)$. Now, we prove estimate (13). The proof is the same as in the first case except for the right-hand side term which is treated as follows:

$$
\int_{0}^{1} f(t, x) u(t, x) d x \leq\|u(t, \cdot)\|_{L^{2}(0,1)}\|f(t, \cdot)\|_{L^{2}(0,1)} .
$$

When integrating between $t$ and $T$, we have

$$
\int_{t}^{T} \int_{0}^{1} f u d x d s \leq\|u\|_{L^{\infty}\left(0, T ; L^{2}(0,1)\right)}\|f\|_{L^{1}\left(0, T ; L^{2}(0,1)\right)} .
$$

Using Young's inequality $a b \leq \varepsilon a^{2}+\varepsilon^{-1} b^{2}$ and taking into account that the term $\|u\|_{L^{\infty}\left(0, T ; L^{2}(0,1)\right)}^{2}$ is produced by the left hand side of (17), we obtain estimate (13). 


\subsubsection{The case where $M$ is variable}

Here we prove that there exists a positive constant $\tilde{C}$ such that

$$
\|u\|_{L^{\infty}\left(0, T ; L^{2}(0,1)\right)}+\|u\|_{L^{2}\left(0, T ; H^{1}(0,1)\right)}+\left\|u_{x \mid x=1}\right\|_{L^{2}(0, T)} \leq \tilde{C}\left(\|M\|_{Y_{1 / 4}}, \nu\right)\|f\|_{L^{2}\left(0, T ; H^{-1}(0,1)\right)},
$$

and

$$
\|u\|_{L^{\infty}\left(0, T ; L^{2}(0,1)\right)}+\|u\|_{L^{2}\left(0, T ; H^{1}(0,1)\right)}+\left\|u_{x \mid x=1}\right\|_{L^{2}(0, T)} \leq \tilde{C}\left(\|M\|_{Y_{1 / 4}}, \nu\right)\|f\|_{L^{1}\left(0, T ; L^{2}(0,1)\right)} .
$$

The analysis is the same in both situations $f \in L^{2}\left(0, T ; H^{-1}(0,1)\right)$ and $f \in L^{1}\left(0, T ; L^{2}(0,1)\right)$, so we will only sketch the proof of the first one. When we multiply equation $(9)$ by $(1-x) u$, we estimate the term concerning $M$ in the following way:

$$
\left|-\int_{0}^{1}(1-x) M u u_{x} d x\right| \leq \frac{\nu}{2} \int_{0}^{1}\left|u_{x}\right|^{2} d x+(C / \nu)\|M(t)\|_{L^{\infty}(0,1)}^{2} \int_{0}^{1}|u|^{2} d x .
$$

When we multiply the equation of $u$ by $u$, we also have estimate (20) for the term concerning $M$. From the corresponding inequalities (15) and (16), we obtain

$$
\begin{aligned}
& -\frac{1}{2} \frac{d}{d t} \int_{0}^{1}(2-x)|u|^{2} d x+\frac{\nu}{2} \int_{0}^{1}\left|u_{x}\right|^{2} d x+\frac{\nu}{2}\left|u_{x \mid x=1}\right|^{2} \\
& \leq C\left(1+(1 / \nu)\|M(t)\|_{L^{\infty}(0,1)}^{2}\right) \int_{0}^{1}|u|^{2} d x+(C / \nu)\|f(t)\|_{H^{-1}(0,1)}^{2} .
\end{aligned}
$$

Using Gronwall's lemma and thanks to the assumption $M \in L^{2}\left(0, T ; H^{1}(0,1)\right)$, we obtain (18) and (19).

\subsection{Additional regularity estimate for $M=0$}

Here we prove an additional regularity result for the following system:

$$
\begin{cases}-u_{t}-\nu u_{x x x}=g & \text { in }(0, T) \times(0,1), \\ u_{\mid x=0}=u_{\mid x=1}=u_{x \mid x=0}=0 & \text { in }(0, T) \\ u_{\mid t=T}=0 & \text { in }(0,1) .\end{cases}
$$

Let us introduce some functional spaces which will be useful in the sequel:

$$
\begin{gathered}
X_{0}:=L^{2}\left(0, T ; H^{-2}(0,1)\right), \quad X_{1}:=L^{2}\left(0, T ; H_{0}^{2}(0,1)\right), \\
\widetilde{X}_{0}:=L^{1}\left(0, T ; H^{-1}(0,1)\right), \quad \widetilde{X}_{1}:=L^{1}\left(0, T ;\left(H^{3} \cap H_{0}^{2}\right)(0,1)\right), \\
Y_{0}:=L^{2}((0, T) \times(0,1)) \cap C^{0}\left([0, T] ; H^{-1}(0,1)\right),
\end{gathered}
$$

and

$$
Y_{1}:=L^{2}\left(0, T ; H^{4}(0,1)\right) \cap C^{0}\left([0, T] ; H^{3}(0,1)\right) .
$$

The spaces $X_{0}$ and $X_{1}$ are equipped with their natural norms, while $Y_{0}$ and $Y_{1}$ are equipped with

$$
\|w\|_{Y_{0}}:=\nu^{1 / 2}\|w\|_{L^{2}((0, T) \times(0,1))}+\|w\|_{L^{\infty}\left(0, T ; H^{-1}(0,1)\right)},
$$

and

respectively.

$$
\|w\|_{Y_{1}}:=\nu^{1 / 2}\|w\|_{L^{2}\left(0, T ; H^{4}(0,1)\right)}+\|w\|_{L^{\infty}\left(0, T ; H^{3}(0,1)\right)} .
$$

Now, for each $\theta \in[0,1]$ we define the (complex) interpolation spaces

$$
X_{\theta}:=\left(X_{0}, X_{1}\right)_{[\theta]}, \quad \widetilde{X}_{\theta}:=\left(\widetilde{X}_{0}, \widetilde{X}_{1}\right)_{[\theta]}, \quad \text { and } \quad Y_{\theta}:=\left(Y_{0}, Y_{1}\right)_{[\theta]} .
$$

Observe that this notation is consistent with the notations of Proposition 3 and (11).

The regularity result is obtained in two steps:

- First in Paragraph 2.3.1, we prove a regularity result in the space $Y_{1}$,

- Next, in Paragraph 2.3.2, we interpolate this result with the one of Paragraph 2.2.1. 


\subsubsection{Regularity result in $Y_{1}$}

In this paragraph we prove that for $g \in L^{2}\left(0, T ; H_{0}^{2}(0,1)\right) \cup L^{1}\left(0, T ;\left(H^{3} \cap H_{0}^{2}\right)(0,1)\right)$, u belongs to $Y_{1}$ and there exists a positive constant $C$ (independent of $\nu$ ) such that

$$
\|w\|_{Y_{1}}+\nu^{-1 / 2}\left\|w_{x \mid x=1}\right\|_{H^{1}(0, T)} \leq\left(C / \nu^{1 / 2}\right)\|g\|_{L^{2}\left(0, T ; H^{2}(0,1)\right)}
$$

and

$$
\|w\|_{Y_{1}}+\nu^{-1 / 2}\left\|w_{x \mid x=1}\right\|_{H^{1}(0, T)} \leq C\|g\|_{L^{1}\left(0, T ; H^{3}(0,1)\right)} .
$$

We will suppose that $g$ is in $C^{\infty}([0, T] \times[0,1])$ with $g_{\mid x=0}=g_{\mid x=1}=g_{x \mid x=0}=g_{x \mid x=1}=0$. Again, the conclusion in the cases $g \in L^{2}\left(0, T ; H_{0}^{2}(0,1)\right)$ and $g \in L^{1}\left(0, T ;\left(H^{3} \cap H_{0}^{2}\right)(0,1)\right)$ follows from an approximation argument.

Let us apply the operator $P_{1}=\partial_{x x x}$ to equation (22):

$$
\left(P_{1} u\right)_{t}+\nu P_{1}^{2} u=-P_{1} g \quad \text { in }(0, T) \times(0,1) .
$$

We multiply this equation by $-(1-x) P_{1} u$ and we integrate in $(0,1)$ :

$$
-\frac{1}{2} \frac{d}{d t} \int_{0}^{1}(1-x)\left|P_{1} u\right|^{2} d x-\nu \int_{0}^{1}(1-x) P_{1}^{2} u P_{1} u d x=\int_{0}^{1}(1-x) P_{1} u P_{1} g d x .
$$

We compute the second term in the left hand side:

$$
\begin{aligned}
-\nu \int_{0}^{1}(1-x) P_{1}^{2} u P_{1} u d x & =\nu \int_{0}^{1}\left(\partial_{x x}\right)\left(P_{1} u\right)\left((1-x) P_{1} u_{x}-P_{1} u\right) d x \\
& =\frac{3 \nu}{2} \int_{0}^{1}\left|P_{1} u_{x}\right|^{2} d x .
\end{aligned}
$$

Here, we used $P_{1} u_{\mid x=0,1}=P_{1} u_{x \mid x=0}=0$, which comes from (22) and the conditions on the traces of $g$ on the boundaries 0 and 1 .

Let us now multiply equation $(27)$ by $-P_{1} u$ and integrate in $(0,1)$ :

$$
-\frac{1}{2} \frac{d}{d t} \int_{0}^{1}\left|P_{1} u\right|^{2} d x-\nu \int_{0}^{1} P_{1}^{2} u P_{1} u d x=\int_{0}^{1} P_{1} u P_{1} g d x .
$$

The second term gives now:

$$
-\nu \int_{0}^{1} P_{1}^{2} u P_{1} u d x=\frac{\nu}{2} \int_{0}^{1} \partial_{x}\left|P_{1} u_{x}\right|^{2} d x=\frac{1}{2 \nu}\left|u_{x t \mid x=1}\right|^{2} .
$$

The boundary conditions which we just used are $P_{1} u_{\mid x=0,1}=0, P_{1} u_{x \mid x=0}=0$ and $\nu P_{1} u_{x \mid x=1}=-u_{x t \mid x=1}$. As previously, the latter equalities follow from (22) and the conditions on the traces of $g$.

Putting together (28)-(31), we obtain

$$
-\frac{1}{2} \frac{d}{d t} \int_{0}^{1}(2-x)\left|P_{1} u\right|^{2} d x+\frac{3}{2} \nu \int_{0}^{1}\left|P_{1} u_{x}\right|^{2} d x+\frac{1}{2 \nu}\left|u_{x t \mid x=1}\right|^{2}=\int_{0}^{1}(2-x) P_{1} u P_{1} g d x .
$$

Integrating between $t$ and $T$, we get

$$
\begin{aligned}
\frac{1}{2} \int_{0}^{1}(2-x)\left|P_{1} u\right|^{2}(t) d x+\frac{3}{2} \nu \int_{t}^{T} \int_{0}^{1}\left|P_{1} u_{x}\right|^{2} d x d s+\frac{1}{2 \nu} \int_{t}^{T}\left|u_{x t \mid x=1}\right|^{2} d s & \\
& =\int_{t}^{T} \int_{0}^{1}(2-x) P_{1} u P_{1} g d x d s
\end{aligned}
$$

for a. e. $t \in(0, T)$. Now, to estimate the last term in (33), we distinguish the two functional frameworks for $g$ : 
- First case: $g \in L^{2}\left(0, T ; H_{0}^{2}(0,1)\right)$. Using $P_{1} u_{\mid x=0,1}=0$ and integrating by parts we get:

$$
\int_{0}^{T} \int_{0}^{1}(2-x) P_{1} u P_{1} g d x d s=-\int_{0}^{T} \int_{0}^{1} g_{x x}\left((2-x) P_{1} u_{x}-P_{1} u\right) d x d s .
$$

Hence we deduce

$$
\begin{aligned}
\left|\int_{0}^{T} \int_{0}^{1}(2-x) P_{1} u P_{1} g d x d s\right| & \leq C\left\|P_{1} u\right\|_{L^{2}\left(0, T ; H^{1}(0,1)\right)}\left\|g_{x x}\right\|_{L^{2}\left(0, T ; L^{2}(0,1)\right)} \\
& \leq \frac{\nu}{2}\left\|P_{1} u\right\|_{L^{2}\left(0, T ; H^{1}(0,1)\right)}^{2}+\frac{C}{\nu}\left\|g_{x x}\right\|_{L^{2}((0, T) \times(0,1))}^{2} .
\end{aligned}
$$

- Second case: $g \in L^{1}\left(0, T ;\left(H^{3} \cap H_{0}^{2}\right)(0,1)\right)$. In that case the estimate is more direct:

$$
\begin{aligned}
\left|\int_{0}^{T} \int_{0}^{1}(2-x) P_{1} u P_{1} g d x d s\right| & \leq 2\left\|P_{1} u\right\|_{L^{\infty}\left(0, T ; L^{2}(0,1)\right)}\left\|P_{1} g\right\|_{L^{1}\left(0, T ; L^{2}(0,1)\right)} \\
& \leq \frac{1}{4}\left\|P_{1} u\right\|_{L^{\infty}\left(0, T ; L^{2}(0,1)\right)}^{2}+4\left\|P_{1} g\right\|_{L^{1}\left(0, T ; L^{2}(0,1)\right)}^{2} .
\end{aligned}
$$

Now we inject (34) and (35) in (33) and take the supremum in $t \in(0, T)$. Finally, we use the following Poincaré's inequalities: $\forall v \in H^{4}(0,1)$ such that $v(0)=v(1)=v^{\prime}(0)=0$, one has

$$
\|v\|_{H^{3}(0,1)} \leq C\left\|P_{1} v\right\|_{L^{2}(0,1)} \text { and }\|v\|_{H^{4}(0,1)} \leq C\left\|P_{1} v\right\|_{H^{1}(0,1)} .
$$

Consequently we deduce the desired inequalities (25) and (26).

\subsubsection{Interpolation arguments}

From Paragraphs 2.2.1 and 2.3.1, we can define a linear mapping $A: g \mapsto u$, where $u$ is the solution of (22). This mapping continuously maps $X_{1 / 4}$ and $\widetilde{X}_{1 / 4}$ to $Y_{1 / 4}$, and $X_{1}$ and $\widetilde{X}_{1}$ to $Y_{1}$. Moreover in these various situations, the norm of the operator $A$ can be estimated by (see (12), (13), (25) and (26))

$$
\|A\|_{\mathcal{L}\left(X_{1 / 4} ; Y_{1 / 4}\right)} \leq C / \nu^{1 / 2},\|A\|_{\mathcal{L}\left(\widetilde{X}_{1 / 4} ; Y_{1 / 4}\right)} \leq C,\|A\|_{\mathcal{L}\left(X_{1} ; Y_{1}\right)} \leq C / \nu^{1 / 2} \text { and }\|A\|_{\mathcal{L}\left(\widetilde{X}_{1} ; Y_{1}\right)} \leq C .
$$

From classical interpolation arguments (see e.g. [1]), we have that $A$ continuously maps $X_{\theta}$ and $\widetilde{X}_{\theta}$ to $Y_{\theta}$, for any $\theta \in[1 / 4,1]$. Moreover the corresponding operator norms satisfy

$$
\|A\|_{\mathcal{L}\left(X_{\theta} ; Y_{\theta}\right)} \leq C / \nu^{1 / 2} \text { and }\|A\|_{\mathcal{L}\left(\widetilde{X}_{\theta} ; Y_{\theta}\right)} \leq C .
$$

In the same manner, we can define a linear operator $B: g \mapsto u_{x \mid x=1}$, which continuously maps $X_{1 / 4}$ and $\widetilde{X}_{1 / 4}$ to $L^{2}(0, T)$ and $X_{1}$ and $\widetilde{X}_{1}$ to $H^{1}(0, T)$. The same interpolation argument yields for $\theta \in[1 / 4,1]$ :

$$
\|B\|_{\mathcal{L}\left(X_{\theta} ; H^{\frac{4}{3}(\theta-1 / 4)}(0, T)\right)} \leq C \nu^{\frac{4}{3}(\theta-1)} \text { and }\|B\|_{\mathcal{L}\left(\tilde{X}_{\theta} ; H^{\frac{4}{3}(\theta-1 / 4)}(0, T)\right)} \leq C \nu^{\frac{4}{3}\left(\theta-\frac{5}{8}\right)} .
$$

Taking $\theta=1 / 2$, we obtain that:

- If $g \in X_{1 / 2}=L^{2}((0, T) \times(0,1))$ then the solution of (22) satisfies $u \in Y_{1 / 2}$ and $u_{x \mid x=1} \in H^{1 / 3}(0, T)$, with

$$
\|u\|_{Y_{1 / 2}}+\nu^{1 / 6}\left\|u_{x \mid x=1}\right\|_{H^{1 / 3}(0, T)} \leq\left(C / \nu^{1 / 2}\right)\|g\|_{X_{1 / 2}} .
$$

- If $g \in \widetilde{X}_{1 / 2}=L^{1}\left(0, T ; H_{0}^{1}(0,1)\right)$ then the solution of (22) also satisfies $u \in Y_{1 / 2}$ and $u_{x \mid x=1} \in H^{1 / 3}(0, T)$, with

$$
\|u\|_{Y_{1 / 2}}+\nu^{1 / 6}\left\|u_{x \mid x=1}\right\|_{H^{1 / 3}(0, T)} \leq C\|g\|_{\widetilde{X}_{1 / 2}} .
$$

Observe that, in this case, we have

$$
Y_{1 / 2}=L^{2}\left(0, T ; H^{2}(0,1)\right) \cap C^{0}\left([0, T] ; H^{1}(0,1)\right) .
$$


This already yields $u_{\mid t=0} \in H^{1}(0,1)$ and $u_{x \mid x=1} \in H^{1 / 3}(0, T)$ with

$$
\left\|u_{\mid t=0}\right\|_{H^{1}(0,1)} \leq\left\{\begin{array}{l}
\left(C / \nu^{1 / 2}\right)\|g\|_{X_{1 / 2}} \\
C\|g\|_{\widetilde{X}_{1 / 2}}
\end{array}\right.
$$

and

$$
\left\|u_{x \mid x=1}\right\|_{H^{1 / 3}(0, T)} \leq\left\{\begin{array}{l}
\left(C / \nu^{2 / 3}\right)\|g\|_{X_{1 / 2}} \\
\left(C / \nu^{1 / 6}\right)\|g\|_{\tilde{X}_{1 / 2}}
\end{array} .\right.
$$

It only remains to prove the property in (10) which concerns the terms $u_{x x \mid x=0}$ and $u_{x x \mid x=1}$. Let us prove, for instance, an estimate for $u_{x x \mid x=0}$ (the same can be done for $u_{x x \mid x=1}$ ). For this, we introduce $\rho \in C^{3}([0,1])$ satisfying

$$
\rho_{\mid[0,1 / 2]}=1 \quad \text { and } \quad \rho_{\mid[3 / 4,1]}=0 .
$$

Let us consider the function $\widehat{u}=\rho(x) u$, which fulfills the equation

$$
\widehat{u}_{t}+\nu \widehat{u}_{x x x}=\nu\left(3 \rho_{x} u_{x x}+3 \rho_{x x} u_{x}+\rho_{x x x} u\right)-\rho(x) g .
$$

Multiplying this equation by $-\widehat{u}_{x x}$ and integrating in $(0,1)$, we obtain:

$$
\frac{1}{2} \frac{d}{d t} \int_{0}^{1}\left|\widehat{u}_{x}\right|^{2} d x+\frac{\nu}{2}\left|u_{x x \mid x=0}\right|^{2}=-\int_{0}^{1}\left(\nu\left(3 \rho_{x} u_{x x}+3 \rho_{x x} u_{x}+\rho_{x x x} u\right)-\rho g\right) \widehat{u}_{x x} d x .
$$

Integrating now in the $t$ variable and estimating the right hand side terms, we deduce

$$
\frac{\nu}{2} \int_{0}^{T}\left|u_{x x \mid x=0}\right|^{2} d t \leq C\left(\nu\|u\|_{L^{2}\left(0, T ; H^{2}(0,1)\right)}^{2}+\|u\|_{L^{\infty}\left(0, T ; H^{1}(0,1)\right)}^{2}\right)+\left|\int_{0}^{T} \int_{0}^{1} \rho g \widehat{u}_{x x} d x d t\right| .
$$

Let us distinguish both situations in order to bound the last integral in the right hand side of (44):

- If $g \in X_{1 / 2}$ we simply use Cauchy-Schwarz inequality:

$$
\left|\int_{0}^{T} \int_{0}^{1} \rho g \widehat{u}_{x x} d x d t\right| \leq 2\left(\nu^{-1}\|g\|_{L^{2}((0, T) \times(0,1))}^{2}+\nu\|u\|_{L^{2}\left(0, T ; H^{2}(0,1)\right)}^{2}\right) .
$$

- If $g \in \widetilde{X}_{1 / 2}$ we integrate by parts once more in the $x$ variable:

$$
\left|\int_{0}^{T} \int_{0}^{1} \rho g \widehat{u}_{x x} d x d t\right|=\left|\int_{0}^{T} \int_{0}^{1} \widehat{u}_{x}\left(\rho_{x} g+\rho g_{x}\right) d x d t\right| \leq C\left(\|g\|_{L^{1}\left(0, T ; H^{1}(0,1)\right)}^{2}+\|u\|_{L^{\infty}\left(0, T ; H^{1}(0,1)\right)}^{2}\right) .
$$

Then, from (44), we deduce in both situations that

$$
\left\|u_{x x \mid x=0}\right\|_{L^{2}(0, T)}^{2} \lesssim\|u\|_{L^{2}\left(0, T ; H^{2}(0,1)\right)}^{2}+\nu^{-1}\|u\|_{L^{\infty}\left(0, T ; H^{1}(0,1)\right)}^{2}+\left\{\begin{array}{l}
\nu^{-2}\|g\|_{L^{2}((0, T) \times(0,1))}^{2}, \\
\nu^{-1}\|g\|_{L^{1}\left(0, T, H^{1}(0,1)\right)}^{2} .
\end{array}\right.
$$

Thanks to (39) and (40), we finally deduce that

$$
\nu^{-1}\|u\|_{Y_{1 / 2}}^{2}+\left\|u_{x x \mid x=0}\right\|_{L^{2}(0, T)}^{2} \leq\left\{\begin{array}{l}
\left(C / \nu^{2}\right)\|g\|_{L^{2}((0, T) \times(0,1))}^{2} \\
(C / \nu)\|g\|_{L^{1}\left(0, T, H^{1}(0,1)\right)}^{2}
\end{array}\right.
$$

Combining (41) and (42)-(46), we obtain

$$
\begin{aligned}
\left\|u_{\mid t=0}\right\|_{H^{1}(0,1)} & +\nu^{1 / 6}\left\|u_{x \mid x=1}\right\|_{H^{1 / 3}(0, T)} \\
& +\nu^{1 / 2}\left\|u_{x x \mid x=0}\right\|_{L^{2}(0, T)}+\nu^{1 / 2}\left\|u_{x x \mid x=1}\right\|_{L^{2}(0, T)} \leq\left\{\begin{array}{l}
\left(C / \nu^{1 / 2}\right)\|g\|_{L^{2}((0, T) \times(0,1))}, \\
C\|g\|_{L^{1}\left(0, T, H^{1}(0,1)\right)} .
\end{array}\right.
\end{aligned}
$$




\subsection{Conclusion}

We begin by noticing that the solution $u$ of (9) also solves $(22)$ when $g:=f-M(t, x) u_{x}$. When $f \in L^{2}((0, T) \times(0,1))$ we directly apply the first inequality in $(47)$, whereas when $f \in L^{1}\left(0, T ; H_{0}^{1}(0,1)\right)$ we decompose $u=u_{1}+u_{2}$ where $u_{1}$ satisfies (22) with $g=f$ and $u_{2}$ satisfies (22) with $g=-M(t, x) u_{x}$. Thus, we get

$$
\begin{aligned}
\left\|u_{\mid t=0}\right\|_{H^{1}(0,1)}+\nu^{1 / 6}\left\|u_{x \mid x=1}\right\|_{H^{1 / 3}(0, T)}+\nu^{1 / 2}\left\|u_{x x \mid x=0}\right\|_{L^{2}(0, T)} \\
+\nu^{1 / 2}\left\|u_{x x \mid x=1}\right\|_{L^{2}(0, T)} \leq\left\{\begin{array}{l}
\left(C / \nu^{1 / 2}\right)\left\|f-M u_{x}\right\|_{L^{2}((0, T) \times(0,1))}, \\
C\|f\|_{L^{1}\left(0, T, H^{1}(0,1)\right)}+\left(C / \nu^{1 / 2}\right)\left\|M u_{x}\right\|_{L^{2}((0, T) \times(0,1))} .
\end{array}\right.
\end{aligned}
$$

Now we distinguish the cases where $M$ is constant and where $M$ is variable:

- $M$ is constant: in order to estimate the $M u_{x}$ term, we use (13):

$$
\begin{array}{r}
\left\|u_{\mid t=0}\right\|_{H^{1}(0,1)}+\nu^{1 / 6}\left\|u_{x \mid x=1}\right\|_{H^{1 / 3}(0, T)}+\nu^{1 / 2}\left\|u_{x x \mid x=0}\right\|_{L^{2}(0, T)} \\
+\nu^{1 / 2}\left\|u_{x x \mid x=1}\right\|_{L^{2}(0, T)} \leq\left\{\begin{array}{l}
(C / \nu)\|f\|_{L^{2}((0, T) \times(0,1))}, \\
(C / \nu)\|f\|_{L^{1}\left(0, T, H^{1}(0,1)\right)} .
\end{array}\right.
\end{array}
$$

- $M$ is variable: we estimate the right hand side with

$$
\begin{aligned}
\left\|M u_{x}\right\|_{L^{2}((0, T) \times(0,1))} & \leq\|M\|_{L^{\infty}\left(0, T ; L^{2}(0,1)\right)}\left\|u_{x}\right\|_{L^{2}\left(0, T ; L^{\infty}(0,1)\right)} \\
& \leq C\|M\|_{L^{\infty}\left(0, T ; L^{2}(0,1)\right)}\|u\|_{L^{2}\left(0, T ; H^{7 / 4}(0,1)\right)} .
\end{aligned}
$$

Then we use that for any $\delta>0$, there exists $C_{\delta}>0$ such that

$$
\|u\|_{L^{2}\left(0, T ; H^{7 / 4}(0,1)\right)} \leq \delta\|u\|_{L^{2}\left(0, T ; H^{2}(0,1)\right)}+C_{\delta}\|u\|_{L^{2}\left(0, T ; H^{1}(0,1)\right)} .
$$

Finally, using the energy estimates (18) and (19), we get:

$$
\begin{aligned}
&\left\|u_{\mid t=0}\right\|_{H^{1}(0,1)}+\left\|u_{x \mid x=1}\right\|_{H^{1 / 3}(0, T)} \\
&+\left\|u_{x x \mid x=0}\right\|_{L^{2}(0, T)}+\left\|u_{x x \mid x=1}\right\|_{L^{2}(0, T)} \leq\left\{\begin{array}{l}
\widetilde{C}\left(\nu,\|M\|_{Y_{1 / 4}}\right)\|f\|_{L^{2}((0, T) \times(0,1))}, \\
\widetilde{C}\left(\nu,\|M\|_{Y_{1 / 4}}\right)\|f\|_{L^{1}\left(0, T, H^{1}(0,1)\right)} .
\end{array}\right.
\end{aligned}
$$

Now, the conclusions of Proposition 1, 2 and 3 are consequences of Riesz Theorem and Definition 1 (see identity (8)). Observe that the continuity in time can be obtained from the $L^{\infty}$ regularity and a classical density argument since for smooth data we actually have the continuity in time.

\section{Proofs of Theorems 2 and 3}

\subsection{Carleman inequality}

We recall that here $M$ is a constant. Let us consider the following backwards (in time) problem, which is usually called the adjoint system associated to (1):

$$
\begin{cases}-\varphi_{t}-\nu \varphi_{x x x}-M \varphi_{x}=0 & \text { in }(0, T) \times(0,1), \\ \varphi_{\mid x=0}=\varphi_{\mid x=1}=\varphi_{x \mid x=0}=0 & \text { in }(0, T), \\ \varphi_{\mid t=T}=\varphi_{0} & \text { in }(0,1) .\end{cases}
$$

The objective of this paragraph is to prove a Carleman inequality for the solutions of this system. After a simple change of time variable in (52), we have

$$
\begin{cases}-\varphi_{t}-\varphi_{x x x}-(M / \nu) \varphi_{x}=0 & \text { in }\left(0, T_{0}\right) \times(0,1):=Q_{0}, \\ \varphi_{\mid x=0}=\varphi_{\mid x=1}=\varphi_{x \mid x=0}=0 & \text { in }\left(0, T_{0}\right), \\ \varphi_{\mid t=T_{0}}=\varphi_{0} & \text { in }(0,1),\end{cases}
$$


where $T_{0}:=\nu T$. We will rather work with this equation for which obtaining a Carleman inequality will be clearer. In order to state this estimate, let us set

$$
\alpha(t, x)=\frac{100+4 x-x^{2}}{t^{1 / 2}\left(T_{0}-t\right)^{1 / 2}}
$$

for $(t, x) \in Q_{0}$. Weight functions of this kind were first introduced by A. V. Fursikov and O. Yu. Imanuvilov; see [9] for a systematic use of them. We denote

$$
\check{\alpha}(t):=\min _{x \in[0,1]} \alpha(t, x)=\alpha(t, 0) \text { and } \hat{\alpha}(t):=\max _{x \in[0,1]} \alpha(t, x)=\alpha(t, 1) .
$$

Observe that the function $\alpha$ satisfies

$$
\begin{gathered}
C \leq T_{0} \alpha, \quad C_{0} \alpha \leq \alpha_{x} \leq C_{1} \alpha, \quad C_{0} \alpha \leq-\alpha_{x x} \leq C_{1} \alpha \quad \text { in }\left(0, T_{0}\right) \times[0,1] \\
\left|\alpha_{t}\right|+\left|\alpha_{x t}\right|+\left|\alpha_{x x t}\right| \leq C T_{0} \alpha^{3}, \quad\left|\alpha_{t t}\right| \leq C\left(T_{0}^{2} \alpha^{5}+\alpha^{3}\right) \leq C T_{0}^{2} \alpha^{5} \quad \text { in }\left(0, T_{0}\right) \times[0,1]
\end{gathered}
$$

and

$$
64 \check{\alpha}-62 \hat{\alpha}=\frac{14}{\left(t\left(T_{0}-t\right)\right)^{1 / 2}}>0,
$$

where $C, C_{0}$ and $C_{1}$ are positive constants independent of $T_{0}$.

We have:

Proposition 4. There exists a positive constant $C$ independent of $T_{0}, \nu$ and $M$ such that, for any $\varphi_{0} \in L^{2}(0,1)$, we have

$$
\iint_{Q_{0}} \alpha e^{-2 s \alpha}\left(\left|\varphi_{x x}\right|^{2}+s^{2} \alpha^{2}\left|\varphi_{x}\right|^{2}+s^{4} \alpha^{4}|\varphi|^{2}\right) d x d t \leq C \int_{0}^{T_{0}} \alpha_{\mid x=0} e^{-2 s \alpha_{\mid x=0}}\left|\varphi_{x x \mid x=0}\right|^{2} d t
$$

for any $s \geq C\left(T_{0}+T_{0}^{1 / 2}+T_{0}|M|^{1 / 2} / \nu^{1 / 2}\right)$, where $\varphi$ is the solution of (53).

Remark 11. Observe that in (59), the right hand side is bounded: it suffices to apply (10) to the function $\alpha_{\mid x=0}(t)^{1 / 2} e^{-s \alpha_{\mid x=0}(t)} \varphi(t, x)$.

Remark 12. Note that (somewhat different) Carleman estimates for the linear KdV equation have also been obtained by L. Rosier [16, 17].

Since the proof of Proposition 4 is very technical, we postpone it to an appendix, at the end of the paper.

\subsection{Proof of Theorem 2}

Let us first deduce an observability inequality from the Carleman inequality (59). We consider $\varphi$ the solution of (52). By the change of variable $t \rightarrow \nu t$ (recall that $T_{0}=\nu T$ ), we can associate a solution of (53), on which one can apply (59). Thanks to the definition of the weight $\alpha$ (see (54)), we obtain that

$$
s^{2} \iint_{Q_{0}} \alpha^{3} e^{-2 s \alpha}\left|\varphi_{x}\right|^{2} d x d t \leq C \int_{0}^{T_{0}} \alpha(t, 0) e^{-2 s \alpha(t, 0)}\left|\varphi_{x x}(t, 0)\right|^{2} d t,
$$

for some $C>0$ independent of $T_{0}, \nu$ and $M$. From the definition of $\alpha$ (see (54)), this yields

$$
\frac{s^{2}}{T_{0}^{3}} e^{-C_{2} s / T_{0}} \iint_{Q_{0}}\left|\varphi_{x}\right|^{2} d x d t \leq \frac{C_{3}}{T_{0}} e^{-C_{3} s / T_{0}} \int_{0}^{T_{0}}\left|\varphi_{x x}(t, 0)\right|^{2} d t
$$

for some $C_{2}, C_{3}>0$. Let us now establish that

$$
\int_{0}^{1}\left|\varphi_{x}\left(t_{1}, x\right)\right|^{2} d x \leq \frac{C}{\nu^{2 / 3}} \int_{0}^{1}\left|\varphi_{x}\left(t_{2}, x\right)\right|^{2} d x \quad 0 \leq t_{1} \leq t_{2} \leq T
$$


for some $C>0$. We prove (61) by showing the following two estimates:

$$
\int_{0}^{1}\left|\varphi\left(t_{1}, x\right)\right|^{2} d x \leq C \int_{0}^{1}\left|\varphi\left(t_{2}, x\right)\right|^{2} d x
$$

and

$$
\int_{0}^{1}\left|\varphi_{x x x}\left(t_{1}, x\right)\right|^{2} d x \leq \frac{C}{\nu^{2}} \int_{0}^{1}\left|\varphi_{x x x}\left(t_{2}, x\right)\right|^{2} d x
$$

for some $C>0$. Once (62) and (63) are established, we define the operator which maps $\varphi\left(t_{2}\right)$ to $\varphi\left(t_{1}\right)$. Estimate $(62)$ gives the continuity from $L^{2}(0,1)$ to itself, while estimate $(63)$ gives the continuity from

$$
\left\{u \in H^{3}(0,1): u(0)=u(1)=u_{x}(0)=0\right\}
$$

to itself. Then a classical interpolation argument gives the desired estimate (61), that is to say, the continuity from $H_{0}^{1}$ to itself.

First, (62) follows from (17) with $f=0$ which, as can be easily seen, is valid regardless of the initial state $\varphi_{0}$, and by integrating in time. Next, we turn to (63). We define the operator $P_{2}:=\nu \partial_{x x x}^{3}+M \partial_{x}$, we multiply the equation $(52)$ by $-P_{2} \varphi_{t}$ and integrate with respect to $x$ :

$$
\nu \int_{0}^{1} \varphi_{x t} \varphi_{x x t} d x-\frac{1}{2} \frac{d}{d t} \int_{0}^{1}\left|P_{2} \varphi\right|^{2} d x=0,
$$

where we used the boundary condition $\varphi_{\mid x=0,1}=0$. It follows that:

$$
\int_{0}^{1}\left|P_{2} \varphi\left(t_{1}, x\right)\right|^{2} d x \leq \int_{0}^{1}\left|P_{2} \varphi\left(t_{2}, x\right)\right|^{2} d x
$$

Using

$$
\int_{0}^{1}\left|\nu \varphi_{x x x}\left(t_{1}, x\right)\right|^{2} d x \leq 2 \int_{0}^{1}\left|P_{2} \varphi\left(t_{1}, x\right)\right|^{2} d x+2 \int_{0}^{1}\left|M \varphi_{x}\left(t_{1}, x\right)\right|^{2} d x
$$

and

$$
\int_{0}^{1}\left|M \varphi_{x}\left(t_{1}, x\right)\right|^{2} d x \leq \frac{\nu^{2}}{4} \int_{0}^{1}\left|\varphi_{x x x}\left(t_{1}, x\right)\right|^{2} d x+\frac{C}{\nu^{2}} \int_{0}^{1}\left|\varphi\left(t_{1}, x\right)\right|^{2} d x,
$$

we deduce with (64) that

$$
\begin{aligned}
\int_{0}^{1}\left|\varphi_{x x x}\left(t_{1}, x\right)\right|^{2} d x & \leq \frac{C}{\nu^{2}}\left(\int_{0}^{1}\left|P_{2} \varphi\left(t_{2}, x\right)\right|^{2} d x+\int_{0}^{1}\left|\varphi\left(t_{1}, x\right)\right|^{2} d x\right) \\
& \leq \frac{C}{\nu^{2}}\left(\int_{0}^{1}\left|\varphi_{x x x}\left(t_{2}, x\right)\right|^{2} d x+\int_{0}^{1}\left|\varphi\left(t_{1}, x\right)\right|^{2} d x\right) .
\end{aligned}
$$

Using (62) and the first Poincaré's inequality in (36), we deduce (63) and hence (61).

In particular, (61) allows us to deduce the following observability inequality from (60):

$$
\int_{0}^{1}\left|\varphi_{x}(0, x)\right|^{2} d x \leq C^{*} \int_{0}^{T}\left|\varphi_{x x \mid x=0}\right|^{2} d t
$$

with

$$
C^{*}=\exp \left\{\frac{C_{4}|M|^{1 / 2}}{\nu^{1 / 2}}\left(1+\frac{1}{T^{1 / 2}|M|^{1 / 2}}\right)\right\},
$$

for some $C_{4}>0$ which depends on $T$ and for $\nu$ sufficiently small in terms of $M$.

Now, from this observability inequality for the solutions of (52), it is classical to prove that for any $y_{0} \in H^{-1}(0,1)$, there exists a control $v_{1} \in L^{2}(0, T)$ such that the solution $y \in Y_{0}$ of $(1)$ with $v_{2}=v_{3}=0$ satisfies $y(T, x)=0$ for $x \in(0,1)$ with $v_{1}$ estimated by $(3)$. (We recall that the space $Y_{0}$ was defined in (23).) In the case of internal controllability, an explicit construction of this control is made in Section 4. This concludes the proof of Theorem 2. 


\subsection{Proof of Theorem 3}

Let $y_{1} \in L^{2}(0,1)$. We must find two controls $v_{1}$ and $v_{2}$ in $L^{2}(0, T)$ such that the solution $y \in Y_{0}=$ $L^{2}((0, T) \times(0,1)) \cap C^{0}\left([0, T] ; H^{-1}(0,1)\right)$ of the system:

$$
\begin{cases}y_{t}+\nu y_{x x x}+M y_{x}=0 & \text { in }(0, T) \times(0,1), \\ y_{\mid x=0}=v_{1}, y_{\mid x=1}=v_{2}, y_{x \mid x=1}=0 & \text { in }(0, T) \\ y_{\mid t=0}=y_{0} & \text { in }(0,1)\end{cases}
$$

satisfies

$$
y_{\mid t=T}=y_{1} \text { in }(0,1)
$$

We divide the proof in two steps:

- First, we prove that there exists $\widetilde{y}_{0} \in L^{2}(0,1)$ and controls $\widetilde{v}_{1}$ and $\widetilde{v}_{2}$ in $L^{2}(0, T)$ such that the solution $\widetilde{y}$ of

$$
\begin{cases}\widetilde{y}_{t}+\nu \widetilde{y}_{x x x}+M \widetilde{y}_{x}=0 & \text { in }(0, T) \times(0,1), \\ \widetilde{y}_{\mid x=0}=\widetilde{v}_{1}, \widetilde{y}_{\mid x=1}=\widetilde{v}_{2}, \widetilde{y}_{x \mid x=1}=0 & \text { in }(0, T), \\ \widetilde{y}_{\mid t=0}=\widetilde{y}_{0} & \text { in }(0,1)\end{cases}
$$

belongs to $\in Y_{1 / 4}=L^{2}\left(0, T ; H^{1}(0,1)\right) \cap C^{0}\left([0, T] ; L^{2}(0,1)\right)$ and satisfies

$$
\widetilde{y}_{\mid t=T}=y_{1} \text { in }(0,1) \text {. }
$$

Indeed, let $h \in Y_{0}$ be the solution of

$$
\begin{cases}h_{t}+\nu h_{x x x}+M h_{x}=0 & \text { in }(0, T) \times(0,1), \\ h_{\mid x=0}=0, h_{\mid x=1}=0, h_{x \mid x=0}=0 & \text { in }(0, T), \\ h_{\mid t=T}=y_{1, x} & \text { in }(0,1)\end{cases}
$$

in the sense described in Definition 1 (observe that $y_{1, x} \in H^{-1}(0,1)$ ). The existence and regularity of $h$ are provided by Propositions 1 and 2. Let us now introduce

$$
z(t, x):=\int h(t, \xi) d \xi \in Y_{1 / 4}
$$

which is of course determined up to a constant. We define:

$$
\begin{cases}c:=z_{t}+\nu z_{x x x}+M z_{x} & \text { in } \mathcal{D}^{\prime}((0, T) \times(0,1)), \\ d:=y_{1}-z_{\mid t=T} & \text { in } L^{2}(0,1) .\end{cases}
$$

It is a direct consequence of (70) and (71) that

$$
c_{x}=0 \text { and } d_{x}=0,
$$

that is, $c$ is a distribution of time and $d$ is a constant. But from (70), we know that $h_{t}, h_{x x x} \in$ $L^{2}\left(0, T ; H^{-3}(0,1)\right)$, hence $z_{t}, z_{x x x} \in L^{2}\left(0, T ; H^{-2}(0,1)\right)$, so we have $c \in L^{2}(0, T)$. Let us now introduce

$$
g(t)=d+\int_{t}^{T} c(\tau) d \tau
$$

Then, the function $\widetilde{y}(t, x)=z(t, x)+g(t) \in Y_{1 / 4}$ satisfies

$$
\begin{cases}\widetilde{y}_{t}+\nu \widetilde{y}_{x x x}+M \widetilde{y}_{x}=0 & \text { in }(0, T) \times(0,1), \\ \widetilde{y}_{x \mid x=1}=0 & \text { in }(0, T), \\ \widetilde{y}_{\mid t=0}=\widetilde{y}_{0} & \text { in }(0,1)\end{cases}
$$

with $\widetilde{y}_{0}:=z_{\mid t=0}+g(0) \in L^{2}(0,1)$. Note that from $\widetilde{y} \in Y_{1 / 4}$, we deduce that $\widetilde{v}_{1}:=\widetilde{y}_{\mid x=0}$ and $\widetilde{v}_{2}:=\widetilde{y}_{\mid x=1}$ belong to $L^{2}(0, T)$. With these controls, $\widetilde{y}$ satisfies $(68)$ and $(69)$. 
- Then, we apply Theorem 1 for the initial condition $y_{0}-\tilde{y}_{0} \in L^{2}(0,1)$. This yields that there exists a control $\widehat{v}_{1} \in L^{2}(0, T)$ such that the solution $\widehat{y} \in Y_{0}$ of

$$
\begin{cases}\widehat{y}_{t}+\nu \widehat{y}_{x x x}+M \widehat{y}_{x}=0 & \text { in }(0, T) \times(0,1), \\ \widehat{y}_{\mid x=0}=\widehat{v}_{1}, \widehat{y}_{\mid x=1}=0, \widehat{y}_{x \mid x=1}=0 & \text { in }(0, T), \\ \widehat{y}_{\mid t=0}=\widehat{y}_{0}:=y_{0}-\widetilde{y}_{0} & \text { in }(0,1)\end{cases}
$$

satisfies $\widehat{y}_{\mid t=T}=0$ in $(0,1)$.

Finally, defining $y:=\widetilde{y}-\widehat{y}$, we realize that $y \in Y_{0}$ fulfills (66) and satisfies (67).

\section{Controllability of the nonlinear system}

\subsection{Proof of Theorem 4}

\subsubsection{Modified Carleman inequality}

Let us briefly explain why Proposition 4 is still valid when $M$ is replaced by a function $M=M(t, x)$ with $L_{t}^{\infty}\left(L_{x}^{2}\right)$ regularity, that is, when (53) is replaced by

$$
\begin{cases}-\varphi_{t}-\varphi_{x x x}-(M(t, x) / \nu) \varphi_{x}=0 & \text { in }\left(0, T_{0}\right) \times(0,1), \\ \varphi(t, 0)=\varphi(t, 1)=\varphi_{x}(t, 0)=0 & \text { in }\left(0, T_{0}\right), \\ \varphi\left(T_{0}, x\right)=\varphi_{0}(x) & \text { in }(0,1) .\end{cases}
$$

We recall that $T_{0}:=\nu T$.

Proposition 5. There exist two positive constants $C$ and $K\left(T_{0}, \nu,\|M\|_{L^{\infty}\left(0, T ; L^{2}(0,1)\right)}\right)$ such that, for any $\varphi_{0} \in L^{2}(0,1)$, we have

$$
\iint_{Q_{0}} \alpha e^{-2 s \alpha}\left(\left|\varphi_{x x}\right|^{2}+s^{2} \alpha^{2}\left|\varphi_{x}\right|^{2}+s^{4} \alpha^{4}|\varphi|^{2}\right) d x d t \leq C \int_{0}^{T_{0}} \alpha_{\mid x=0} e^{-2 s \alpha_{\mid x=0}}\left|\varphi_{x x \mid x=0}\right|^{2} d t,
$$

for any $s \geq K$, where $\varphi$ is the solution of (75).

The proof of this proposition will be given at the end of the paper, in Paragraph 6.2.

\subsubsection{Linear controllability with more regular controls}

Let $z \in Y_{1 / 4}$. We consider the following linear control system:

$$
\begin{cases}w_{t}+((1+\bar{y}+z / 2) w)_{x}+\nu w_{x x x}=0 & \text { in }(0, T) \times(0,1), \\ w_{\mid x=0}=v_{1}, w_{\mid x=1}=0, w_{x \mid x=1}=0 & \text { in }(0, T), \\ w_{\mid t=0}=w_{0} & \text { in }(0,1),\end{cases}
$$

where $w_{0}$ is some state in $H^{1}(0,1)$ with $w_{0}(1)=0$.

- Boundary observability inequality. From the Carleman inequality (76) with $M(t, x)=1+\bar{y}(t, x)+$ $z(t, x) / 2$ and taking into account the same analysis developed in Paragraph 3.2, we deduce the existence of a positive constant $C^{*}$ such that

$$
\int_{0}^{1}|\varphi(0, \cdot)|^{2} d x \leq C^{*}\left(T_{0}, \nu,\|M\|_{Z}\right) \int_{0}^{T}\left|\varphi_{x x \mid x=0}\right|^{2} d t
$$

From the observability inequality (78), it is classical to deduce that equation (77) is null controllable with a control $v_{1} \in L^{2}(0, T)$. But in the sequel, it will be convenient to have a control which is more regular than $L^{2}(0, T)$ (in order to perform a fixed point argument (see paragraph 4.1.3) below). 
- An interior control problem. For that purpose, we will consider an internal controllability problem. Let us introduce a linear extension operator $\Pi_{1}$, which maps functions on $[0,1]$ to functions on $[-1,1]$ with support in $[-1 / 2,1]$, and which is continuous from $L^{2}(0,1)$ to $L^{2}(-1,1)$ and from $H^{1}(0,1)$ to $H^{1}(-1,1)$. We define

$$
\widetilde{w}_{0}:=\Pi_{1}\left(w_{0}\right) \text { in } H^{1}(-1,1)
$$

and

$$
\widetilde{\bar{y}}:=\Pi_{1}(\bar{y}) \text { and } \widetilde{z}:=\Pi_{1}(z) \text { in } L^{2}\left(0, T ; H^{1}(-1,1)\right) \cap L^{\infty}\left(0, T ; L^{2}(-1,1)\right) .
$$

Let $\omega$ be some interval $(-1,-1+a)$ for some $0<a<1$. The controllability problem under view is the following:

$$
\begin{cases}\widetilde{w}_{t}+((1+\widetilde{\bar{y}}+\widetilde{z} / 2) \widetilde{w})_{x}+\nu \widetilde{w}_{x x x}=v(t, x) \mathbf{1}_{\omega}(x) & \text { in }(0, T) \times(-1,1), \\ \widetilde{w}_{\mid x=-1}=0, \widetilde{w}_{\mid x=1}=0, \widetilde{w}_{x \mid x=1}=0 & \text { in }(0, T), \\ \widetilde{w}_{\mid t=0}=\widetilde{w}_{0} & \text { in }(-1,1) .\end{cases}
$$

Let us prove that there exists $v \in L^{2}((0, T) \times \omega)$ such that the solution of $(81)$ satisfies

$$
\widetilde{w}_{\mid t=T}=0 \text { in }(-1,1) \text {. }
$$

- Interior observability inequality. For this, we consider the adjoint system associated to (81) with $T_{0}=\nu T:$

$$
\begin{cases}-\phi_{t}-\phi_{x x x}-((1+\widetilde{\bar{y}}+\widetilde{z} / 2) / \nu) \phi_{x}=0 & \text { in }\left(0, T_{0}\right) \times(-1,1), \\ \phi_{\mid x=-1}=\phi_{\mid x=1}=\phi_{x \mid x=-1}=0 & \text { in }\left(0, T_{0}\right), \\ \phi\left(T_{0}, x\right)=\phi_{0}(x) & \text { in }(-1,1) .\end{cases}
$$

Of course, by performing the change of variable $x \rightarrow 2 x-1$, one can transform (76) into:

$$
\begin{array}{r}
\iint_{\left(0, T_{0}\right) \times(-1,1)} \alpha\left(t, \frac{1+x}{2}\right) e^{-2 s \alpha\left(t, \frac{1+x}{2}\right)}\left(\left|\phi_{x x}\right|^{2}+s^{2} \alpha\left(t, \frac{1+x}{2}\right)^{2}\left|\phi_{x}\right|^{2}+s^{4} \alpha\left(t, \frac{1+x}{2}\right)^{4}|\phi|^{2}\right) d x d t \\
\leq C_{5} \int_{0}^{T_{0}} \alpha_{\mid x=0} e^{-2 s \alpha_{\mid x=0}\left|\phi_{x x \mid x=-1}\right|^{2} d t}
\end{array}
$$

for some $C_{5}>0$. It is straightforward from (83) that

$$
\iint_{\left(0, T_{0}\right) \times(-1,1)} \check{\alpha} e^{-2 s \hat{\alpha}}\left(\left|\phi_{x x}\right|^{2}+s^{2} \check{\alpha}^{2}\left|\phi_{x}\right|^{2}+s^{4} \check{\alpha}^{4}|\phi|^{2}\right) d x d t \leq C_{5} \int_{0}^{T_{0}} \check{\alpha} e^{-2 s \check{\alpha}}\left|\phi_{x x \mid x=-1}\right|^{2} d t .
$$

Recall that $\hat{\alpha}$ and $\check{\alpha}$ are given by $(55)$.

To reach the interior observability result which we seek, we transform this inequality in two steps: first we modify the right-hand side, and next we modify the left-hand side.

- Right-hand side. We have

$$
\begin{aligned}
\int_{0}^{T_{0}} \check{\alpha} e^{-2 s \check{\alpha}}\left|\phi_{x x \mid x=-1}\right|^{2} d t & \leq C \int_{0}^{T_{0}} \check{\alpha} e^{-2 s \check{\alpha}}\|\phi(t, \cdot)\|_{H^{31 / 12}(\omega)}^{2} d t \\
& \leq C \int_{0}^{T_{0}} \check{\alpha} e^{-2 s \check{\alpha}}\|\phi(t, \cdot)\|_{H^{8 / 3}(\omega)}^{31 / 16}\|\phi(t, \cdot)\|_{L^{2}(\omega)}^{1 / 16} d t
\end{aligned}
$$

where we have successively used: a trace inequality (observe that $5 / 2<31 / 12$ ) and the interpolation inequality corresponding to $H^{31 / 12}$ interpolated between $H^{8 / 3}$ and $L^{2}$. Now we use Young's inequality to get

$$
\begin{aligned}
\int_{0}^{T_{0}} \check{\alpha} e^{-2 s \check{\alpha}}\left|\phi_{x x \mid x=-1}\right|^{2} d t \\
\quad \leq C \int_{0}^{T_{0}} e^{-(31 / 16) s \hat{\alpha}} \check{\alpha}^{-279 / 32}\|\phi(t, \cdot)\|_{H^{8 / 3}(\omega)}^{31 / 16} e^{-2 s \check{\alpha}} e^{(31 / 16) s \hat{\alpha}} \check{\alpha}^{311 / 32}\|\phi(t, \cdot)\|_{L^{2}(\omega)}^{1 / 16} d t \\
\leq \varepsilon \int_{0}^{T_{0}} e^{-2 s \hat{\alpha}} \check{\alpha}^{-9}\|\phi(t, \cdot)\|_{H^{8 / 3}(\omega)}^{2} d t+C_{\varepsilon} \int_{0}^{T_{0}} e^{s(-64 \check{\alpha}+62 \hat{\alpha})} \check{\alpha}^{311}\|\phi(t, \cdot)\|_{L^{2}(\omega)}^{2} d t
\end{aligned}
$$


where $\varepsilon$ is to be fixed later.

- Left hand side. We introduce

$$
\phi_{1}(t, x):=\theta_{1}(t) \phi(t, x)
$$

with

$$
\theta_{1}(t)=\exp (-s \hat{\alpha}) \check{\alpha}^{-1 / 2} .
$$

Now, $\phi_{1}$ satisfies the following system

$$
\left\{\begin{array}{l}
-\phi_{1 t}-\phi_{1 x x x}=g_{1}, \\
\phi_{1 \mid x=-1}=\phi_{1 \mid x=1}=\phi_{1_{x \mid x=-1}}=0, \\
\phi_{1}\left(T_{0}, x\right)=0,
\end{array}\right.
$$

where

$$
g_{1}=((1+\widetilde{\bar{y}}+\widetilde{z} / 2) / \nu) \theta_{1} \phi_{x}-\theta_{1 t} \phi .
$$

Now we estimate $\phi_{1}$ in terms of the $L^{2}\left(\left(0, T_{0}\right) \times(-1,1)\right)$-norm of the right hand side of (86). By employing estimate (39) and recalling (41), we have in particular $\phi_{1} \in L^{4}\left(0, T_{0} ;\left(H^{3 / 2} \cap H_{0}^{1}\right)(-1,1)\right)$ and

$$
\left\|\phi_{1}\right\|_{L^{4}\left(0, T_{0} ; H^{3 / 2}(-1,1)\right)} \leq C\left\|g_{1}\right\|_{L^{2}\left(\left(0, T_{0}\right) \times(-1,1)\right)},
$$

for some $C>0$. As $\widetilde{\bar{y}}, \widetilde{z} \in L^{\infty}\left(0, T_{0} ; L^{2}(-1,1)\right)$ and $\left|\theta_{1 t}\right| \leq C \check{\alpha}^{5 / 2} \exp (-s \hat{\alpha})$, we have

$$
\left\|g_{1}\right\|_{L^{2}\left(\left(0, T_{0}\right) \times(-1,1)\right)}^{2} \leq C \iint_{\left(0, T_{0}\right) \times(-1,1)} \check{\alpha} e^{-2 s \hat{\alpha}}\left(\left|\phi_{x x}\right|^{2}+s^{4} \check{\alpha}^{4}|\phi|^{2}\right) d x d t .
$$

Now we define

$$
\phi_{2}(t, x):=\theta_{2}(t) \phi(t, x),
$$

with

$$
\theta_{2}(t)=\exp (-s \hat{\alpha}) \check{\alpha}^{-5 / 2} .
$$

It follows that $\phi_{2}$ satisfies (86), with $g_{1}$ replaced by

$$
g_{2}:=((1+\widetilde{\bar{y}}+\widetilde{z} / 2) / \nu) \theta_{2} \theta_{1}^{-1} \phi_{1 x}-\theta_{2 t} \theta_{1}^{-1} \phi_{1} .
$$

Interpolating (18) and (25), we have $\phi_{2} \in L^{2}\left(0, T_{0} ; H^{7 / 3}(-1,1)\right) \cap L^{\infty}\left(0, T_{0} ; H^{4 / 3}(-1,1)\right)$ and

$$
\left\|\phi_{2}\right\|_{L^{2}\left(0, T_{0} ; H^{7 / 3}(-1,1)\right) \cap L^{\infty}\left(0, T_{0} ; H^{4 / 3}(-1,1)\right)} \leq C\left\|g_{2}\right\|_{L^{2}\left(0, T_{0} ; H^{1 / 3}(-1,1)\right)} .
$$

Observing that $\theta_{2} \theta_{1}^{-1}$ and $\theta_{2 t} \theta_{1}^{-1}$ are bounded and $\widetilde{\bar{y}}, \widetilde{z} \in L^{4}\left(0, T_{0} ; H^{1 / 2}(-1,1)\right.$ ) (as easily seen by interpolation), we find that

$$
\left\|g_{2}\right\|_{L^{2}\left(0, T_{0} ; H^{1 / 3}(-1,1)\right)} \leq C\left\|\phi_{1}\right\|_{L^{4}\left(0, T_{0} ; H^{3 / 2}(-1,1)\right)} .
$$

Here, we have also used that the product of two $H^{1 / 2}(-1,1)$ functions belongs to $H^{1 / 3}(-1,1)$.

Finally, we define $\phi_{3}:=\theta_{3}(t) \phi(t, x)$ with

$$
\theta_{3}(t)=\exp (-s \hat{\alpha}) \check{\alpha}^{-9 / 2} .
$$

We have again that $\phi_{3}$ satisfies (86) with, in place of $g_{1}$ :

$$
g_{3}:=((1+\widetilde{\bar{y}}+\widetilde{z} / 2) / \nu) \theta_{3} \theta_{2}^{-1} \phi_{2 x}-\theta_{3 t} \theta_{2}^{-1} \phi_{2} .
$$

Using the same arguments as previously and the fact that $\widetilde{y}$ and $\widetilde{z}$ are bounded in $L^{3}\left(0, T ; H^{2 / 3}(-1,1)\right)$, we arrive at

$$
\left\|\phi_{3}\right\|_{L^{2}\left(0, T_{0} ; H^{8 / 3}(-1,1)\right) \cap L^{\infty}\left(0, T_{0} ; H^{5 / 3}(-1,1)\right)} \leq C\left\|g_{3}\right\|_{L^{2}\left(0, T_{0} ; H^{2 / 3}(-1,1)\right)},
$$

and

$$
\left\|g_{3}\right\|_{L^{2}\left(0, T_{0} ; H^{2 / 3}(-1,1)\right)} \leq C\left\|\phi_{2}\right\|_{L^{6}\left(0, T_{0} ; H^{5 / 3}(-1,1)\right)} .
$$


Finally, putting together (87)-(88), (90)-(91) and (93)-(94), we obtain

$$
\left\|\phi_{3}\right\|_{L^{2}\left(0, T_{0} ; H^{8 / 3}(-1,1)\right)}^{2} \leq C_{6} \iint_{\left(0, T_{0}\right) \times(-1,1)} \check{\alpha} e^{-2 s \hat{\alpha}}\left(\left|\phi_{x x}\right|^{2}+s^{4} \check{\alpha}^{4}|\phi|^{2}\right) d x d t,
$$

for some $C_{6}>0$. We now express (95) in terms of $\phi$ :

$$
\int_{0}^{T_{0}} e^{-2 s \hat{\alpha}} \check{\alpha}^{-9}\|\phi(t, \cdot)\|_{H^{8 / 3}(-1,1)}^{2} d t \leq C_{6} \iint_{\left(0, T_{0}\right) \times(-1,1)} \check{\alpha} e^{-2 s \hat{\alpha}}\left(\left|\phi_{x x}\right|^{2}+s^{4} \check{\alpha}^{4}|\phi|^{2}\right) d x d t .
$$

Lastly, we fix $\varepsilon=\left(2 C_{5} C_{6}\right)^{-1}$ in (85) and using (84), it results:

$$
\begin{aligned}
\iint_{\left(0, T_{0}\right) \times(-1,1)} \check{\alpha} e^{-2 s \hat{\alpha}}\left(\left|\phi_{x x}\right|^{2}+s^{2} \check{\alpha}^{2}\left|\phi_{x}\right|^{2}+s^{4} \check{\alpha}^{4}|\phi|^{2}\right) & d x d t \\
& \leq C \int_{0}^{T_{0}} e^{s(-64 \check{\alpha}+62 \hat{\alpha})} \check{\alpha}^{311}\|\phi(t, \cdot)\|_{L^{2}(\omega)}^{2} d t .
\end{aligned}
$$

Recalling (58) and using again Paragraph 3.2, we reach the following interior observability inequality:

$$
\int_{-1}^{1}|\phi(0, \cdot)|^{2} d x \leq C_{*} \int_{0}^{T}\|\phi(t, \cdot)\|_{L^{2}(\omega)}^{2} d t
$$

- Design of the control. From (98), we can deduce the existence of $v(t) \in L^{2}((0, T) \times \omega)$ answering the null controllability problem by using the following method, which will help us to single out a particular control; this will be useful when handling the nonlinear problem. On $L^{2}(-1,1)$ we introduce the following norm: $\left\|\phi_{0}\right\|_{F}:=\|\phi\|_{L^{2}((0, T) \times \omega)}$, where $\phi$ is the solution of (82) associated to $\phi_{0}$. The fact that this is a norm comes from the unique continuation property for system (82) which follows for instance from (98).

Let $F$ be the space obtained by completing $L^{2}(-1,1)$ with the above norm. We define $J$ as the following functional on $F$ :

$$
J\left(\phi_{0}\right):=\frac{1}{2}\left\|\phi_{0}\right\|_{F}^{2}+\int_{-1}^{1} \phi(0, x) \widetilde{w}_{0}(x) d x .
$$

The fact that the second term is well-defined on $F$ and that it is continuous as a function of $\phi_{0}$ is a consequence of (98). Since $J$ is moreover strictly convex and coercive (as follows again from (98)), the functional $J$ admits a unique minimum $\phi_{0}^{\star}$, which furthermore is characterized by the following Euler-Lagrange equation:

$$
\forall \chi_{0} \in F, \quad \iint_{(0, T) \times \omega} \chi \phi^{\star} d x d t+\int_{-1}^{1} \chi(0, x) \widetilde{w}_{0}(x) d x=0,
$$

where again $\chi$ and $\phi^{\star}$ are the solutions of (82) associated to $\chi_{0}$ and $\phi_{0}^{\star}$, respectively. Now, we define $v \in L^{2}((0, T) \times \omega)$ by

$$
v:=\mathbf{1}_{\omega} \phi^{\star}
$$

Hence for any $\phi_{0} \in L^{2}(-1,1)$ we have

$$
\int_{-1}^{1} \widetilde{w}(T, x) \phi_{0} d x=\iint_{(0, T) \times(-1,1)} v \phi d x d t+\int_{-1}^{1} \phi(0, x) \widetilde{w}_{0}(x) d x=0,
$$

where $\widetilde{w}$ is the solution of (81) associated to $v$ defined in (100). Hence $v$ is a control which steers $\widetilde{w}_{0}$ to 0 . Its norm can be estimated by setting $\chi=\phi^{\star}$ in (99); with (98) this yields

$$
\begin{aligned}
\|v\|_{L^{2}((0, T) \times \omega)} & \leq C\left\|\widetilde{w}_{0}\right\|_{L^{2}(-1,1)} \\
& \leq C^{\prime}\left\|w_{0}\right\|_{L^{2}(0,1)}
\end{aligned}
$$

Thanks to estimate (39), we deduce that $\widetilde{w} \in Y_{1 / 2}$ and

$$
\|\widetilde{w}\|_{Y_{1 / 2}} \leq C\left(\|v\|_{L^{2}((0, T) \times \omega)}+\left\|\widetilde{w}_{0}\right\|_{H^{1}(-1,1)}\right),
$$


for some $C>0$. Observe that estimate (39) was established for $u$ solution of (9), which has a null initial condition but the general situation follows directly from our proof.

- Back to the boundary control problem. Let

$$
w:=\widetilde{w}_{\mid(0, T) \times[0,1]} .
$$

Then, $w$ solves system (77) for

$$
v_{1}:=\widetilde{w}_{\mid x=0} .
$$

We clearly have that $w(T, \cdot)=0$ in $(0,1)$. Furthermore, from (101), (102), equation (77) and interpolation arguments we get that $v_{1} \in H^{(1 / 2)-\varepsilon}(0, T)$ for every $\varepsilon>0$ and

$$
\left\|v_{1}\right\|_{H^{(1 / 2)-\varepsilon}(0, T)} \leq C\left\|w_{0}\right\|_{H^{1}(0,1)},
$$

for some $C>0$.

\subsubsection{Fixed point argument}

Let us recall that $y$ and $\bar{y}$ fulfill systems (5) and (4), respectively. Then, $p=y-\bar{y}$ satisfies:

$$
\begin{cases}p_{t}+p_{x}+p p_{x}+(\bar{y} p)_{x}+\nu p_{x x x}=0 & \text { in }(0, T) \times(0,1), \\ p_{\mid x=0}=v_{1}, p_{\mid x=1}=0, p_{x \mid x=1}=0 & \text { in }(0, T), \\ p_{\mid t=0}=p_{0}:=y_{0}-\bar{y}_{0} & \text { in }(0,1) .\end{cases}
$$

Our objective is to find $v_{1}$ such that the solution of $(106)$ satisfies $p(T, \cdot)=0$.

Remark 13. In the sequel, we will suppose that $p_{0} \in H^{1}(0,1)$ and that $\left\|p_{0}\right\|_{H^{1}(0,1)}$ is sufficiently small. Observe that this can always be assumed by taking $v_{1}=0$ during some time, taking into account the regularizing effect of (106) and using the fact that $\left\|p_{0}\right\|_{L^{2}(0,1)}$ is sufficiently small.

Then, let us introduce the following fixed point mapping. First we introduce the space

$$
E_{0}:=C^{0}\left([0, T] ; L^{2}(0,1)\right) \cap L^{2}\left(0, T ; H^{1}(0,1)\right) \cap H^{1}\left(0, T ; H^{-2}(0,1)\right) .
$$

We consider in $L^{2}((0, T) \times(0,1))$ the following compact subset:

$$
B:=\left\{z \in E_{0} /\|z\|_{E_{0}} \leq 1\right\}
$$

To any $z \in B$, we will associate a set of solutions $w$ of $(77)$ with initial condition $w_{0}=p_{0}$ given in the previous paragraph. More precisely, let us first define the set of controls:

$$
A(z):=\left\{v \in L^{2}((0, T) \times \omega) / \widetilde{w} \text { solution of }(81) \text { satisfies } \widetilde{w}_{\mid t=T}=0 \text { and } v \text { satisfies }(101)\right\} .
$$

We define

$$
\Lambda_{0}(z):=\left\{w:=\widetilde{w}_{\mid(0, T) \times[0,1]} / \widetilde{w} \text { fulfills system }(81) \text { for some control } v \in A(z)\right\} .
$$

Of course, in the above definition, the function $\tilde{w}$ satisfies (81) in the sense of Definition 1 , with the functions $\widetilde{w}_{0}, \widetilde{\bar{y}}$ and $\widetilde{z}$ appearing in (81) defined by (79)-(80).

We will use the following Banach space version of Kakutani's fixed point theorem (see, for instance, [20, Theorem 9.2.3]):

Theorem 6. Let $Z$ be a Banach space and let $\Lambda_{0}: B \rightarrow 2^{B}$ be a set-valued mapping satisfying the following assumptions:

1. $\Lambda_{0}(z)$ is a nonempty closed convex set of $Z$ for every $z \in B$,

2. $\Lambda_{0}$ maps $B$ to a compact subset of $B$, 
3. the graph of $\Lambda_{0}$

$$
\mathcal{G}\left(\Lambda_{0}\right)=\left\{(x, y) \in B \times B / y \in \Lambda_{0}(x)\right\},
$$

is closed in $Z^{2}$.

Then $\Lambda_{0}$ possesses a fixed point in the set $B$, i.e. there exists $z \in B$ such that $z \in \Lambda_{0}(z)$.

Let us check that Theorem 6 can be applied to $\Lambda_{0}$ and

$$
Z=L^{2}((0, T) \times(0,1)) .
$$

- The fact that $\Lambda_{0}(z)$ is a nonempty closed convex set of $Z$ for every $z \in B$ is very easy to verify, so we leave it to the reader.

- That $B$ is a compact subset of $Z$ is easily seen by Rellich's theorem and interpolation arguments.

- Let us observe that provided that $p_{0}$ is small enough, $\Lambda_{0}$ maps $B$ into $2^{B}$. Thanks to (101) and (102) we have that for each $z \in B \subset Z$ the solution $w$ of (81) belongs to $E_{0}$ and there exists a constant $C>0$ such that

$$
\|w\|_{E_{0}} \leq C\left\|p_{0}\right\|_{H^{1}(0,1)} \leq 1 .
$$

The last inequality is obtained by taking $\left\|p_{0}\right\|_{H^{1}(0,1)}$ sufficiently small.

- It remains to check that the graph of $\Lambda_{0}$ is closed. Consider $\left(z_{n}, y_{n}\right)$ a sequence converging in $\left[L^{2}((0, T) \times(0,1))\right]^{2}$ to $(z, y)$, with $y_{n} \in \Lambda_{0}\left(z_{n}\right)$. We have to prove that $y \in \Lambda_{0}(z)$. It is sufficient to be able to pass to the limit in each term of

$$
\widetilde{y_{n}}+\left(\left(1+\widetilde{\bar{y}}+\widetilde{z_{n}} / 2\right) \widetilde{y_{n}}\right)_{x}+\nu \widetilde{y_{n x x}}=v_{n}(t, x) \mathbf{1}_{\omega}(x) \text { in }(0, T) \times(-1,1) .
$$

Note that $v_{n}$ is bounded in $L^{2}$, and hence converges weakly to $v$, up to a subsequence. The only non-trivial convergence is the one of the nonlinear term $\widetilde{z_{n}} \widetilde{y_{n}}$. But, up to a subsequence, using the compactness of $B, \widetilde{y_{n}}$ converges to $\widetilde{y}$ weakly in $L^{2}\left(0, T ; H^{1}(-1,1)\right)$. As $\widetilde{z_{n}}$ converges strongly in $L^{2}((0, T) \times(-1,1))$, it follows that $\widetilde{z_{n}} \widetilde{y_{n}}$ weakly converges to $z y_{x}$.

This shows that $y \in \Lambda_{0}(z)$ and, therefore, the graph of $\Lambda_{0}$ is closed.

Consequently, Theorem 6 applies and this implies that there exists $p \in \Lambda_{0}(p)$, that is to say, we have found a control $v_{1} \in H^{1 / 2-\varepsilon}(0, T)$ for all $\varepsilon>0$, such that the solution solution of (106) satisfies $p(T, \cdot)=0$ in $(0,1)$. The proof of Theorem 4 is finished.

\subsection{Proof of Theorem 5}

This part is close to [15]. First, we introduce the operator $L_{0}: L^{2}(0,1) \rightarrow L^{2}(0,1)$ which associates to any $y_{1} \in L^{2}(0,1)$ the function $\widetilde{y}_{0} \in L^{2}(0,1)$ constructed in Paragraph 3.3 in order to fulfill (68)-(69) with $M=1$.

Next, we introduce the operator $L_{1}: L^{2}(0,1) \rightarrow L^{2}(0, T)$ which associates to any $\widehat{y}_{0} \in L^{2}(0,1)$ the control $v_{1} \in H^{1 / 2-\varepsilon}(0, T)$ constructed in Paragraph 4.1.2 in the case $z=\bar{y}=0$ : see (104). We call $\widehat{L}_{1}: L^{2}(0,1) \rightarrow L^{2}\left(0, T ; H^{1}(0,1)\right) \cap C^{0}\left([0, T] ; L^{2}(0,1)\right)$ the operator which associates to $\widehat{y}_{0} \in L^{2}(0,1)$ the corresponding solution $w$ given by (103). We underline that $L_{1}$ and $\widehat{L}_{1}$ are linear operators. Indeed, for what concerns the (uniquely defined) control $v$, this follows from the characteristic property (99) and the linearity of $\Pi_{1}$. Remark that the continuity of $L_{1}$ and $\widehat{L}_{1}$ comes from (105).

Finally, we define the operator $L_{2}: L^{1}\left(0, T ; L^{2}(0,1)\right) \rightarrow L^{2}\left(0, T ; H_{0}^{1}(0,1)\right) \cap C^{0}\left([0, T] ; L^{2}(0,1)\right)$ which associates to $f$ the (unique) solution $u$ of

$$
\begin{cases}u_{t}+\nu u_{x x x}+u_{x}=f & \text { in }(0, T) \times(0,1), \\ u_{\mid x=0}=u_{\mid x=1}=u_{x \mid x=1}=0 & \text { in }(0, T), \\ u_{\mid t=0}=0 & \text { in }(0,1) .\end{cases}
$$

Observe that this application is well-defined thanks to (13). 
With all this, we are ready to define our fixed-point mapping $\Lambda_{1}: B(0 ; R) \subset L^{2}\left(0, T ; H^{1}(0,1)\right) \rightarrow$ $L^{2}\left(0, T ; H^{1}(0,1)\right)$, where $R>0$ is to be determined; it is defined as:

$$
\Lambda_{1}(u)=\widehat{L}_{1}\left[y_{0}-L_{0}\left\{y_{1}+L_{2}\left(u u_{x}\right)(T)\right\}\right] .
$$

Note that of course, $u u_{x}=\left(u^{2} / 2\right)_{x}$ belongs to $L^{1}\left(0, T ; L^{2}(0,1)\right)$ when $u \in L^{2}\left(0, T ; H^{1}(0,1)\right)$, hence $\Lambda_{1}$ is well-defined. Let us prove that it maps $B(0 ; R)$ into itself and that it is contractive.

- $\Lambda_{1}$ is contractive. Let $u, v \in B(0 ; R)$. We call $\mathcal{C}_{1}, \mathcal{C}_{2}, \mathcal{C}_{3}$ various constants depending only on the operator norms of the above $L_{1}$, etc. We have

$$
\begin{aligned}
\left\|\Lambda_{1}(u)-\Lambda_{1}(v)\right\|_{L^{2}\left(0, T ; H^{1}(0,1)\right)} & \leq\left\|\widehat{L}_{1} \circ L_{0} \circ\left[L_{2}\left(u u_{x}-v v_{x}\right)\right](T)\right\|_{L^{2}\left(0, T ; H^{1}(0,1)\right)} \\
& \leq \mathcal{C}_{1}\left\|L_{0} \circ\left[L_{2}\left(u u_{x}-v v_{x}\right)\right](T)\right\|_{L^{2}(0,1)} \\
& \left.\leq \mathcal{C}_{2} \| L_{2}\left(u u_{x}-v v_{x}\right)\right] \|_{C^{0}\left([0, T] ; L^{2}(0,1)\right)} \\
& \leq \mathcal{C}_{3}\left\|u^{2}-v^{2}\right\|_{L^{1}\left(0, T ; H^{1}(0,1)\right)} \\
& \leq 2 R \mathcal{C}_{3}\|u-v\|_{L^{2}\left(0, T ; H^{1}(0,1)\right)} .
\end{aligned}
$$

Hence $\Lambda_{1}$ is contractive for $R$ small enough, typically

$$
R<\frac{1}{4 \mathcal{C}_{3}} .
$$

- $\Lambda_{1}$ maps $B(0 ; R)$ into itself. Now consider $u \in B(0 ; R)$, and observe in the same way as previously that

$$
\left\|\Lambda_{1}(u)\right\|_{L^{2}\left(0, T ; H^{1}(0,1)\right)} \leq \mathcal{C}_{1}\left\|y_{0}\right\|_{L^{2}(0,1)}+\mathcal{C}_{2}\left\|y_{1}\right\|_{L^{2}(0,1)}+\mathcal{C}_{3} R^{2} .
$$

Hence with the choice (109) and if $\left\|y_{0}\right\|_{L^{2}(0,1)}$ and $\left\|y_{1}\right\|_{L^{2}(0,1)}$ are small enough, the operator $\Lambda_{1}$ maps $B(0 ; R)$ into itself.

In that case, the operator $\Lambda_{1}$ admits a fixed point, by the Banach-Picard Theorem. Then it is straightforward to see that such a fixed point answers to the requirements of Theorem 5 .

\section{Proof of Theorem 1}

We start the proof of Theorem 1 by showing that one can suppose that the initial condition has null traces at $x=0$ and $x=1$.

This is done as follows. For any $\eta>0$, we introduce a linear continuous extension operator $\Pi_{2}$ from $W^{1, \infty}(0,1)$ to $W_{0}^{1, \infty}(-\eta, 1+\eta)$. Consider the problem

$$
\begin{cases}y_{t}+\nu y_{x x x}+M y_{x}=0 & \text { in }(0, T) \times(-\eta, 1+\eta), \\ y_{\mid x=-\eta}=v_{1}, y_{\mid x=1+\eta}=v_{2}, y_{x \mid x=1+\eta}=v_{3} & \text { in }(0, T), \\ y_{\mid t=0}=\Pi_{2}\left(y_{0}\right) & \text { in }(-\eta, 1+\eta) .\end{cases}
$$

Recall that $M$ is a negative constant in this section. Clearly, by rescaling, this problem is equivalent to

$$
y_{t}+\frac{\nu}{(1+2 \eta)^{3}} y_{x x x}+\frac{M}{(1+2 \eta)} y_{x}=0 \text { in }(0, T) \times(0,1) .
$$

Hence, raising $K_{0}$, diminishing $\nu_{0}$ if necessary and taking $\eta$ sufficiently small, we see that solving the problem with $W_{0}^{1, \infty}$ initial data is sufficient. From now on, we suppose $y_{0} \in W_{0}^{1, \infty}(0,1)$.

Now the proof of Theorem 1 is performed in two times. First we drive the state to a small one, and next we drive it to 0 exactly. 


\subsection{Driving the initial state to a "small" one}

In this paragraph we drive the initial state $y_{0} \in W_{0}^{1, \infty}(0,1)$ to some "small" state. This is only possible as long as the transport term "helps", that is to say, when $M<0$.

Introduce the following initial state associated to $y_{0}$, but defined in the whole real line:

$$
u_{0}(x):= \begin{cases}y_{0}(x) & x \in(0,1) \\ 0 & \text { elsewhere. }\end{cases}
$$

The idea we follow here is to consider the solution $u$ of our system associated to $u_{0}$ in $\mathbb{R}$. Then, we prove that $u$ is "small" in the interval $x \in(0,1)$ as long as a time $t>1 /|M|$ has gone by. Finally, it suffices to take the controls as the corresponding traces of $u$ in order to prove the desired result.

Proposition 6. Consider $u_{0} \in W^{1, \infty}(\mathbb{R})$ given by (111). Let $M<0$ and let $u$ be the solution of

$$
\begin{cases}u_{t}+\nu u_{x x x}+M u_{x}=0 & \text { in } \mathbb{R}^{+} \times \mathbb{R}, \\ u_{\mid t=0}=u_{0} & \text { in } \mathbb{R} .\end{cases}
$$

Then the following holds independently of $\nu$ : for $t>1 /|M|$, the function $u$ satisfies:

$$
\|u(t, \cdot)\|_{L^{\infty}(0,1)} \lesssim \frac{\left\|u_{0}\right\|_{\infty}}{(\nu t)^{1 / 3}} \exp \left(-\frac{2}{3} \frac{(-M t-1)^{3 / 2}}{\sqrt{3 \nu t}}\right) .
$$

Moreover one has

$$
\left\|u_{x}(\cdot, 1)\right\|_{L^{\infty}\left(\mathbb{R}^{+}\right)} \lesssim\left\|u_{0}\right\|_{W^{1, \infty}(\mathbb{R})} .
$$

Proof. By performing the change of variable

$$
v(t, x):=u(t, x+M t),
$$

we arrive at the following equation

$$
v_{t}+\nu v_{x x x}=0,
$$

which has as a fundamental solution

$$
G(t, x)=\frac{1}{(3 \nu t)^{1 / 3}} A i\left(\frac{x}{(3 \nu t)^{1 / 3}}\right),
$$

where $A i$ is the Airy function

$$
A i(x):=\frac{1}{2 \pi} \int_{\operatorname{Im}(\xi)=\eta>0} \exp \left[i\left(x \xi+\frac{\xi^{3}}{3}\right)\right] d \xi .
$$

It follows that for $t>0$,

$$
\begin{aligned}
u(t, x) & =\int_{\mathbb{R}} u_{0}(z) \frac{1}{(3 \nu t)^{1 / 3}} A i\left(\frac{x-M t-z}{(3 \nu t)^{1 / 3}}\right) d z \\
& =\int_{(0,1)} y_{0}(z) \frac{1}{(3 \nu t)^{1 / 3}} A i\left(\frac{x-M t-z}{(3 \nu t)^{1 / 3}}\right) d z .
\end{aligned}
$$

We will use the following lemma:

Lemma 1. The Airy function satisfies the following estimate: for $x>0$ :

$$
0 \leq A i(x) \leq A i(0) \exp \left(-\frac{2}{3} x^{3 / 2}\right)
$$


Proof. It is classical (see for instance [13, p. 214]) that the Airy function is a solution of the following equation:

$$
A i^{\prime \prime}-x A i=0,
$$

with $A i(0)>0$ and $A i^{\prime}(0)<0$. It is then clear that in order to establish (117), it is sufficient to prove that the function $g: x \mapsto \exp \left(-\frac{2}{3} x^{3 / 2}\right)$ satisfies

$$
g^{\prime \prime}-x g \geq 0 \text { for } x>0 .
$$

This is immediate since $g^{\prime \prime}-x g=(1 /(2 \sqrt{x})) g$.

Now going back to the estimate on $u(t, x)$, we get for $x \geq 0$ and $t \geq 1 / M$,

$$
|u(t, x)| \leq \frac{A i(0)\left\|y_{0}\right\|_{\infty}}{(3 \nu t)^{1 / 3}} \exp \left(-\frac{2}{3}\left[\frac{x-M t-1}{(3 \nu t)^{1 / 3}}\right]^{3 / 2}\right),
$$

which yields (113).

To get (114), we first differentiate (116) with respect to $x$, and obtain,

$$
\partial_{x} u(t, x)=\left[\frac{1}{(3 \nu t)^{1 / 3}} A i\left(\frac{\cdot-M t}{(3 \nu t)^{1 / 3}}\right) *\left(u_{0}\right)_{x}\right](x)
$$

and then for $x \geq 1$, using Supp $u_{0} \subset[0,1]$ (recall that $M<0$ ), we have that

$$
\partial_{x} u(t, x)=\left[\frac{1}{(3 \nu t)^{1 / 3}}\left(A i \mathbf{1}_{\mathbb{R}^{+}}\right)\left(\frac{\cdot-M t}{(3 \nu t)^{1 / 3}}\right) *\left(u_{0}\right)_{x}\right](x) .
$$

Denote $A i^{+}:=A i \mathbf{1}_{\mathbb{R}^{+}}(\geq 0)$. One has

$$
\begin{aligned}
\left|\partial_{x} u(t, 1)\right| & \leq\left[\frac{1}{(3 \nu t)^{1 / 3}} A i^{+}\left(\frac{\cdot-M t}{(3 \nu t)^{1 / 3}}\right) *\left|\left(u_{0}\right)_{x}\right|\right](1) \\
& =\int_{\mathbb{R}} \frac{1}{(3 \nu t)^{1 / 3}} A i^{+}\left(\frac{z}{(3 \nu t)^{1 / 3}}\right)\left|\left(u_{0}\right)_{x}(1-M t-z)\right| d z .
\end{aligned}
$$

Noting that $A i^{+} \in L^{1}(\mathbb{R})$ and that

$$
\left\|\frac{1}{(3 \nu t)^{1 / 3}} A i^{+}\left(\frac{\cdot}{(3 \nu t)^{1 / 3}}\right)\right\|_{L^{1}(\mathbb{R})}=\left\|A i^{+}\right\|_{L^{1}(\mathbb{R})} \text { for all } t>0,
$$

we conclude by Young's inequality that

$$
\left|\partial_{x} u(t, 1)\right| \leq\left\|\left(u_{0}\right)_{x}\right\|_{L^{\infty}(0,1)}\left\|A i^{+}\right\|_{L^{1}(\mathbb{R})},
$$

independently of $\nu$.

\subsection{Conclusion}

In this paragraph, we combine the previous paragraph and Section 3 in order to prove the uniform null controllability of (1) (Theorem 1).

Let us consider $y_{0} \in W_{0}^{1, \infty}(0,1)$ and $M<0$.

- Let $T_{1} \in(1 /|M|, T)$ to be chosen later. In the time interval $\left(0, T_{1}\right)$, we consider $u$ the solution of (112) with $u_{0}$ given by (111). Then, it is clear that the solution $y$ of the problem

$$
\begin{cases}y_{t}+\nu y_{x x x}+M y_{x}=0 & \text { in }\left(0, T_{1}\right) \times(0,1), \\ y_{\mid x=0}=u_{\mid x=0}, y_{\mid x=1}=u_{\mid x=1}, y_{x \mid x=1}=u_{x \mid x=1} & \text { in }\left(0, T_{1}\right), \\ y_{\mid t=0}=y_{0} & \text { in }(0,1),\end{cases}
$$

also satisfies (113), that is to say,

$$
\left\|y\left(T_{1}, \cdot\right)\right\|_{L^{\infty}(0,1)} \lesssim \frac{\left\|y_{0}\right\|_{\infty}}{\left(\nu T_{1}\right)^{1 / 3}} \exp \left(-\frac{2}{3} \frac{\left(-M T_{1}-1\right)^{3 / 2}}{\left(3 \nu T_{1}\right)^{1 / 2}}\right) .
$$


- Now, between $t=T_{1}$ and $t=T$, we apply the null controllability result proved in Paragraph 3.2 for the initial condition $y\left(T_{1}, \cdot\right)$ (instead of $\left.y_{0}\right)$. Doing so we obtain

$$
y(T, \cdot)=0 \text { in }(0,1) .
$$

Furthermore, we can require that the control satisfies

$$
\|v\|_{L^{2}\left(T_{1}, T\right)}^{2} \leq \frac{C^{*}}{\nu}\left\|y\left(T_{1}, \cdot\right)\right\|_{H^{-1}(0,1)}^{2},
$$

where $C^{*}$ is the constant in the estimate (3), where we replace $T$ with $T-T_{1}$. It follows that the control satisfies

$$
\begin{aligned}
\|v\|_{L^{2}\left(T_{1}, T\right)}^{2} & \leq \frac{1}{\nu} \exp \left\{\frac{\widetilde{C}|M|^{1 / 2}}{\nu^{1 / 2}}\left(1+\frac{1}{\left(|M|\left(T-T_{1}\right)\right)^{1 / 2}}\right)\right\}\left\|y\left(T_{1}, \cdot\right)\right\|_{L^{2}(0,1)}^{2} \\
& \leq \exp \left\{\frac{C_{7}|M|^{1 / 2}}{\nu^{1 / 2}}\left(1+\frac{1}{\left(|M|\left(T-T_{1}\right)\right)^{1 / 2}}\right)\right\}\left\|y\left(T_{1}, \cdot\right)\right\|_{L^{2}(0,1)}^{2}
\end{aligned}
$$

for $\nu \in\left(0, \nu_{0}\right)$ and some constant $C_{7}>0$. From (121), we get

$\|v\|_{L^{2}\left(T_{1}, T\right)}^{2} \leq \frac{C_{8}}{T_{1}^{2 / 3}} \exp \left\{\frac{1}{\nu^{1 / 2}}\left(C_{8}|M|^{1 / 2}\left(1+\frac{1}{\left(|M|\left(T-T_{1}\right)\right)^{1 / 2}}\right)-\frac{4}{3 \sqrt{3} T_{1}^{1 / 2}}\left(-M T_{1}-1\right)^{3 / 2}\right)\right\}\left\|y_{0}\right\|_{\infty}^{2}$

for $\nu \in\left(0, \nu_{0}\right)$.

Thus, taking $T_{1}>K /|M|$ with $K$ sufficiently large, the previous constant is bounded by

$$
\exp \left\{-C|M|^{1 / 2} / \nu^{1 / 2}\right\} \text { for some } C>0,
$$

when $\nu \in\left(0, \nu_{0}\right)$.

- Hence to finish the proof of Theorem 1, it remains only to prove that the norms

$$
\left\|y_{\mid x=0}\right\|_{L^{2}\left(0, T_{1}\right)},\left\|y_{\mid x=1}\right\|_{L^{2}\left(0, T_{1}\right)},\left\|y_{x \mid x=1}\right\|_{L^{2}\left(0, T_{1}\right)},
$$

are bounded independently of $\nu>0$. This is a direct consequence of (114) for what concerns the control $v_{3}$. For controls $v_{1}$ and $v_{2}$, this is for instance a consequence of the following group estimate for the linear KdV operator:

$$
\sup _{t>0}\|u(t, \cdot)\|_{H^{1}(\mathbb{R})} \leq C\left\|u_{0}\right\|_{H^{1}(\mathbb{R})}
$$

(recall that $u$ fulfills (112)).

\section{Appendix: Proof of Carleman inequality}

\subsection{Proof of Proposition 4}

Let $\psi:=e^{-s \alpha} \varphi$, where $\alpha$ is given by (54) and $\varphi$ fulfills system (53). We deduce that

$$
L_{1} \psi+L_{2} \psi=L_{3} \psi
$$

with

$$
\begin{gathered}
L_{1} \psi=-\psi_{x x x}-\psi_{t}-3 s^{2} \alpha_{x}^{2} \psi_{x}-(M / \nu) \psi_{x} \\
L_{2} \psi=-s^{3} \alpha_{x}^{3} \psi-3 s \alpha_{x} \psi_{x x}-s \alpha_{t} \psi-3 s \alpha_{x x} \psi_{x}-s(M / \nu) \alpha_{x} \psi
\end{gathered}
$$

and

$$
L_{3} \psi=s \alpha_{x x x} \psi+3 s^{2} \alpha_{x} \alpha_{x x} \psi
$$

Then, we have

$$
\left\|L_{1} \psi\right\|_{L^{2}\left(Q_{0}\right)}^{2}+\left\|L_{2} \psi\right\|_{L^{2}\left(Q_{0}\right)}^{2}+2 \iint_{Q_{0}} L_{1} \psi L_{2} \psi d x d t=\left\|L_{3} \psi\right\|_{L^{2}\left(Q_{0}\right)}^{2} .
$$


In the following lines, we will compute the double product term. For the sake of simplicity, let us denote by $\left(L_{i} \psi\right)_{j}(1 \leq i \leq 2,1 \leq j \leq 5)$ the $\mathrm{j}$-th term in the expression of $L_{i} \psi$.

- First, integrating by parts with respect to $x$, we have

$$
\begin{aligned}
\left(\left(L_{1} \psi\right)_{1},\left(L_{2} \psi\right)_{1}\right)_{L^{2}\left(Q_{0}\right)} & =-\frac{s^{3}}{2} \iint_{Q_{0}} \alpha_{x}^{3} \partial_{x}\left|\psi_{x}\right|^{2} d x d t+3 s^{3} \iint_{Q_{0}} \alpha_{x}^{2} \alpha_{x x}\left|\psi_{x}\right|^{2} d x d t \\
& +\frac{3 s^{3}}{2} \iint_{Q_{0}}\left(2 \alpha_{x} \alpha_{x x}^{2}+\alpha_{x}^{2} \alpha_{x x x}\right) \partial_{x}|\psi|^{2} d x d t \\
& =\frac{9 s^{3}}{2} \iint_{Q_{0}} \alpha_{x}^{2} \alpha_{x x}\left|\psi_{x}\right|^{2} d x d t-\frac{s^{3}}{2} \int_{0}^{T_{0}} \alpha_{x \mid x=1}^{3}\left|\psi_{x \mid x=1}\right|^{2} d t \\
& -\frac{3 s^{3}}{2} \iint_{Q_{0}}\left(2 \alpha_{x x}^{3}+6 \alpha_{x} \alpha_{x x} \alpha_{x x x}+\alpha_{x}^{2} \alpha_{4 x}\right)|\psi|^{2} d x d t \\
& \geq \frac{9 s^{3}}{2} \iint_{Q_{0}} \alpha_{x}^{2} \alpha_{x x}\left|\psi_{x}\right|^{2} d x d t-\frac{s^{3}}{2} \int_{0}^{T_{0}} \alpha_{x \mid x=1}^{3}\left|\psi_{x \mid x=1}\right|^{2} d t \\
& -C T_{0}^{2} s^{3} \iint_{Q_{0}} \alpha^{5}|\psi|^{2} d x d t .
\end{aligned}
$$

Here, we have used that $\psi_{\mid x=0,1}=\psi_{x \mid x=0}=0$ and (56).

For the second term, integrating by parts again with respect to $x$, we obtain

$$
\begin{aligned}
\left(\left(L_{1} \psi\right)_{1},\left(L_{2} \psi\right)_{2}\right)_{L^{2}\left(Q_{0}\right)} & =-\frac{3 s}{2} \iint_{Q_{0}} \alpha_{x x}\left|\psi_{x x}\right|^{2} d x d t+\frac{3 s}{2} \int_{0}^{T_{0}} \alpha_{x \mid x=1}\left|\psi_{x x \mid x=1}\right|^{2} d t \\
& -\frac{3 s}{2} \int_{0}^{T_{0}} \alpha_{x \mid x=0}\left|\psi_{x x \mid x=0}\right|^{2} d t \\
& \geq-\frac{3 s}{2} \iint_{Q_{0}} \alpha_{x x}\left|\psi_{x x}\right|^{2} d x d t+\frac{3 s}{2} \int_{0}^{T_{0}} \alpha_{x \mid x=1}\left|\psi_{x x \mid x=1}\right|^{2} d t \\
& -C s \int_{0}^{T_{0}} \alpha_{\mid x=0}\left|\psi_{x x \mid x=0}\right|^{2} d t
\end{aligned}
$$

We consider now the third term of $L_{2} \psi$ and we get

$$
\begin{aligned}
\left(\left(L_{1} \psi\right)_{1},\left(L_{2} \psi\right)_{3}\right)_{L^{2}\left(Q_{0}\right)} & =-\frac{s}{2} \iint_{Q_{0}} \alpha_{t} \partial_{x}\left|\psi_{x}\right|^{2} d x d t+\frac{s}{2} \iint_{Q_{0}} \alpha_{x x t} \partial_{x}|\psi|^{2} d x d t \\
& +\frac{s}{2} \iint_{Q_{0}} \alpha_{x t}\left|\psi_{x}\right|^{2} d x d t=s \iint_{Q_{0}} \alpha_{x t}\left|\psi_{x}\right|^{2} d x d t \\
& -\frac{s}{2} \int_{0}^{T_{0}} \alpha_{t \mid x=1}\left|\psi_{x \mid x=1}\right|^{2} d t-\frac{s}{2} \iint_{Q_{0}} \alpha_{x x x t}|\psi|^{2} d x d t \\
& \geq-C s T_{0}\left(\iint_{Q_{0}} \alpha^{3}\left|\psi_{x}\right|^{2} d x d t+\int_{0}^{T_{0}} \alpha_{\mid x=1}^{3}\left|\psi_{x \mid x=1}\right|^{2} d t\right)
\end{aligned}
$$

thanks to $\alpha_{x x x}=0$ and (56)-(57). 
Furthermore, since $\alpha_{x x x}=0$ and $\psi_{\mid x=0}=\psi_{x \mid x=0}=0$, we have

$$
\begin{aligned}
\left(\left(L_{1} \psi\right)_{1},\left(L_{2} \psi\right)_{4}\right)_{L^{2}\left(Q_{0}\right)} & =-3 s \iint_{Q_{0}} \alpha_{x x}\left|\psi_{x x}\right|^{2} d x d t-\frac{3 s}{2} \iint_{Q_{0}} \alpha_{x x x} \partial_{x}\left|\psi_{x}\right|^{2} d x d t \\
& +3 s \int_{0}^{T_{0}} \alpha_{x x \mid x=1} \psi_{x x \mid x=1} \psi_{x \mid x=1} d t \\
& =-3 s \iint_{Q_{0}} \alpha_{x x}\left|\psi_{x x}\right|^{2} d x d t+\frac{3 s}{2} \iint_{Q_{0}} \alpha_{4 x}\left|\psi_{x}\right|^{2} d x d t \\
& -\frac{3 s}{2} \int_{0}^{T_{0}} \alpha_{x x x \mid x=1}\left|\psi_{x \mid x=1}\right|^{2} d t+3 s \int_{0}^{T_{0}} \alpha_{x x \mid x=1} \psi_{x x \mid x=1} \psi_{x \mid x=1} d t \\
& \geq-3 s \iint_{Q_{0}} \alpha_{x x}\left|\psi_{x x}\right|^{2} d x d t-\frac{1}{2} s \int_{0}^{T_{0}} \alpha_{x \mid x=1}\left|\psi_{x x \mid x=1}\right|^{2} d t . \\
& -C s T_{0}^{2} \int_{0}^{T_{0}} \alpha_{\mid x=1}^{3}\left|\psi_{x \mid x=1}\right|^{2} d t .
\end{aligned}
$$

Observe that in the last term we have used Cauchy-Schwarz inequality.

For the last term of $L_{2} \psi$, we have

$$
\begin{aligned}
\left(\left(L_{1} \psi\right)_{1},\left(L_{2} \psi\right)_{5}\right)_{L^{2}\left(Q_{0}\right)} & =-s(M /(2 \nu)) \iint_{Q_{0}} \alpha_{x} \partial_{x}\left|\psi_{x}\right|^{2} d x d t-s(M / \nu) \iint_{Q_{0}} \alpha_{x x} \psi_{x x} \psi d x d t \\
& =-s(M /(2 \nu)) \int_{0}^{T_{0}} \alpha_{x \mid x=1}\left|\psi_{x \mid x=1}\right|^{2} d t+s(M /(2 \nu)) \iint_{Q_{0}} \alpha_{x x}\left|\psi_{x}\right|^{2} d x d t \\
& -s(M / \nu) \iint_{Q_{0}} \alpha_{x x} \psi_{x x} \psi d x d t
\end{aligned}
$$

Using that $\alpha_{x x}<0$, this yields

$$
\begin{aligned}
\left(\left(L^{1} \psi\right)_{1},\left(L_{2} \psi\right)_{5}\right)_{L^{2}\left(Q_{0}\right)} & \geq-C s|M|\left(T_{0}^{2} / \nu\right)\left(\int_{0}^{T_{0}} \alpha_{\mid x=1}^{3}\left|\psi_{x \mid x=1}\right|^{2} d t+\iint_{Q_{0}} \alpha^{3}\left|\psi_{x}\right|^{2} d x d t\right) \\
& +s \iint_{Q_{0}} \alpha_{x x}\left|\psi_{x x}\right|^{2} d x d t-C s M^{2}\left(T_{0}^{4} / \nu^{2}\right) \iint_{Q_{0}} \alpha^{5}|\psi|^{2} d x d t .
\end{aligned}
$$

All these computations ((126)-(130)) show that

$$
\begin{aligned}
& \left(\left(L_{1} \psi\right)_{1}, L_{2} \psi\right)_{L^{2}\left(Q_{0}\right)} \geq \frac{9 s^{3}}{2} \iint_{Q_{0}} \alpha_{x}^{2} \alpha_{x x}\left|\psi_{x}\right|^{2} d x d t-\frac{7 s}{2} \iint_{Q_{0}} \alpha_{x x}\left|\psi_{x x}\right|^{2} d x d t \\
& -C \iint_{Q_{0}}\left(s T_{0}^{4} M^{2} / \nu^{2}+s^{3} T_{0}^{2}\right) \alpha^{5}|\psi|^{2} d x d t-C s \iint_{Q_{0}}\left(T_{0}+T_{0}^{2}|M| / \nu\right) \alpha^{3}\left|\psi_{x}\right|^{2} d x d t \\
& -\frac{s^{3}}{2} \int_{0}^{T_{0}} \alpha_{x \mid x=1}^{3}\left|\psi_{x \mid x=1}\right|^{2} d t+\frac{s}{2} \int_{0}^{T_{0}} \alpha_{x \mid x=1}\left|\psi_{x x \mid x=1}\right|^{2} d t \\
& -C s \int_{0}^{T_{0}}\left(T_{0}+T_{0}^{2}+T_{0}^{2}|M| / \nu\right) \alpha_{\mid x=1}^{3}\left|\psi_{x \mid x=1}\right|^{2} d t-C s \int_{0}^{T_{0}} \alpha_{x=0}\left|\psi_{x x \mid x=0}\right|^{2} d t
\end{aligned}
$$

- As long as the second term of $L_{1} \psi$ is concerned, we first integrate by parts with respect to $t$ :

$$
\left(\left(L_{1} \psi\right)_{2},\left(L_{2} \psi\right)_{1}\right)_{L^{2}\left(Q_{0}\right)}=-\frac{3 s^{3}}{2} \iint_{Q_{0}} \alpha_{x}^{2} \alpha_{x t}|\psi|^{2} d x d t \geq-C s^{3} T_{0} \iint_{Q_{0}} \alpha^{5}|\psi|^{2} d x d t .
$$


For the second one we use that $\psi_{x \mid t=0}=\psi_{x \mid t=T}=0$ and (57) and we get

$$
\begin{aligned}
\left(\left(L_{1} \psi\right)_{2},\left(L_{2} \psi\right)_{2}\right)_{L^{2}\left(Q_{0}\right)} & =-\frac{3 s}{2} \iint_{Q_{0}} \alpha_{x} \partial_{t}\left|\psi_{x}\right|^{2} d x d t-3 s \iint_{Q_{0}} \alpha_{x x} \psi_{x} \psi_{t} d x d t \\
& =\frac{3 s}{2} \iint_{Q_{0}} \alpha_{x t}\left|\psi_{x}\right|^{2} d x d t-3 s \iint_{Q_{0}} \alpha_{x x} \psi_{x} \psi_{t} d x d t \\
& \geq-C s T_{0} \iint_{Q_{0}} \alpha^{3}\left|\psi_{x}\right|^{2} d^{t} d t-3 s \iint_{Q_{0}} \alpha_{x x} \psi_{x} \psi_{t} d x d t
\end{aligned}
$$

Again using $\psi_{\mid t=0}=\psi_{\mid t=T}=0$ and (57), we deduce

$$
\left(\left(L_{1} \psi\right)_{2},\left(L_{2} \psi\right)_{3}\right)_{L^{2}\left(Q_{0}\right)}=-s \iint_{Q_{0}} \alpha_{t t}|\psi|^{2} d x d t \geq-C s T_{0}^{2} \iint_{Q_{0}} \alpha^{5}|\psi|^{2} d x d t .
$$

Furthermore,

$$
\left(\left(L_{1} \psi\right)_{2},\left(L_{2} \psi\right)_{4}\right)_{L^{2}\left(Q_{0}\right)}=3 s \iint_{Q_{0}} \alpha_{x x} \psi_{x} \psi_{t} d x d t .
$$

Finally, the last product of the second term of $L_{1} \psi$ provides

$$
\left(\left(L_{1} \psi\right)_{2},\left(L_{2} \psi\right)_{5}\right)_{L^{2}\left(Q_{0}\right)}=-s M /(2 \nu) \iint_{Q_{0}} \alpha_{x t}|\psi|^{2} d x d t \geq C s|M|\left(T_{0}^{3} / \nu\right) \iint_{Q_{0}} \alpha^{5}|\psi|^{2} d x d t
$$

Putting together all the computations concerning the second term of $L_{1} \psi((132)-(136))$, we obtain

$$
\begin{aligned}
\left(\left(L_{1} \psi\right)_{2}, L_{2} \psi\right)_{L^{2}\left(Q_{0}\right)} & \geq-C s T_{0}\left(s^{2}+T_{0}+T_{0}^{2}|M| / \nu\right) \iint_{Q_{0}} \alpha^{5}|\psi|^{2} d x d t \\
& -C s T_{0} \iint_{Q_{0}} \alpha^{3}\left|\psi_{x}\right|^{2} d x d t .
\end{aligned}
$$

- We consider now the products concerning the third term of $L_{1} \psi$. First, we have

$$
\left(\left(L_{1} \psi\right)_{3},\left(L_{2} \psi\right)_{1}\right)_{L^{2}\left(Q_{0}\right)}=-\frac{15 s^{5}}{2} \iint_{Q_{0}} \alpha_{x}^{4} \alpha_{x x}|\psi|^{2} d x d t \geq C s^{5} \iint_{Q_{0}} \alpha^{5}|\psi|^{2} d x d t
$$

Secondly,

$$
\left(\left(L_{1} \psi\right)_{3},\left(L_{2} \psi\right)_{2}\right)_{L^{2}\left(Q_{0}\right)}=-\frac{27 s^{3}}{2} \iint_{Q_{0}} \alpha_{x}^{2} \alpha_{x x}\left|\psi_{x}\right|^{2} d x d t+\frac{9 s^{3}}{2} \int_{0}^{T_{0}} \alpha_{x \mid x=1}^{3}\left|\psi_{x \mid x=1}\right|^{2} d t .
$$

Using (57), we obtain the following for the third term:

$$
\begin{aligned}
\left(\left(L_{1} \psi\right)_{3},\left(L_{2} \psi\right)_{3}\right)_{L^{2}\left(Q_{0}\right)} & =-\frac{3 s^{3}}{2} \iint_{Q_{0}}\left(2 \alpha_{x} \alpha_{x x} \alpha_{t}+\alpha_{x}^{2} \alpha_{x t}\right)|\psi|^{2} d x d t \\
& \geq-C s^{3} T_{0} \iint_{Q_{0}} \alpha^{5}|\psi|^{2} d x d t
\end{aligned}
$$

Furthermore,

$$
\left(\left(L_{1} \psi\right)_{3},\left(L_{2} \psi\right)_{4}\right)_{L^{2}\left(Q_{0}\right)}=9 s^{3} \iint_{Q_{0}} \alpha_{x}^{2} \alpha_{x x}\left|\psi_{x}\right|^{2} d x d t
$$

Finally,

$$
\begin{aligned}
\left(\left(L_{1} \psi\right)_{3},\left(L_{2} \psi\right)_{5}\right)_{L^{2}\left(Q_{0}\right)} & =-\frac{9}{2} s^{3}(M / \nu) \iint_{Q_{0}} \alpha_{x}^{2} \alpha_{x x}|\psi|^{2} d x d t \\
& \geq-C s^{3}\left(T_{0}^{2}|M| / \nu\right) \iint_{Q_{0}} \alpha^{5}|\psi|^{2} d x d t
\end{aligned}
$$


Consequently, we get the following for the third term of $L_{1} \psi((138)-(142))$ :

$$
\begin{aligned}
& \left(\left(L_{1} \psi\right)_{3}, L_{2} \psi\right)_{L^{2}\left(Q_{0}\right)} \geq C_{0} s^{5} \iint_{Q_{0}} \alpha^{5}|\psi|^{2} d x d t-\frac{9 s^{3}}{2} \iint_{Q_{0}} \alpha_{x}^{2} \alpha_{x x}\left|\psi_{x}\right|^{2} d x d t \\
& -C s^{3} T_{0}\left(1+T_{0}|M| / \nu\right) \iint_{Q_{0}} \alpha^{5}|\psi|^{2} d x d t+\frac{9 s^{2}}{2} \int_{0}^{T_{0}} \alpha_{x \mid x=1}^{3}\left|\psi_{x \mid x=1}\right|^{2} d t .
\end{aligned}
$$

- As long as the fourth and last term of $L_{1} \psi$ is concerned, we have:

$$
\begin{aligned}
\left(\left(L_{1} \psi\right)_{4},\left(L_{2} \psi\right)_{1}\right)_{L^{2}\left(Q_{0}\right)} & =-\frac{3}{2} s^{3}(M / \nu) \iint_{Q_{0}} \alpha_{x}^{2} \alpha_{x x}|\psi|^{2} d x d t \\
& \geq-C s^{3}|M|\left(T_{0}^{2} / \nu\right) \iint_{Q_{0}} \alpha^{5}|\psi|^{2} d x d t .
\end{aligned}
$$

Now, we compute the second one:

$$
\begin{aligned}
\left(\left(L_{1} \psi\right)_{4},\left(L_{2} \psi\right)_{2}\right)_{L^{2}\left(Q_{0}\right)} & =-\frac{3}{2} s(M / \nu) \iint_{Q_{0}} \alpha_{x x}\left|\psi_{x}\right|^{2} d x d t+\frac{3}{2} s(M / \nu) \int_{0}^{T_{0}} \alpha_{x \mid x=1}\left|\psi_{x \mid x=1}\right|^{2} d t \\
& \geq-C s|M|\left(T_{0}^{2} / \nu\right)\left(\iint_{Q_{0}} \alpha^{3}\left|\psi_{x}\right|^{2} d x d t+\int_{0}^{T_{0}} \alpha_{\mid x=1}^{3}\left|\psi_{x \mid x=1}\right|^{2} d x\right) .
\end{aligned}
$$

Additionally, integrating by parts again with respect to $x$, we have

$\left(\left(L_{1} \psi\right)_{4},\left(L_{2} \psi\right)_{3}\right)_{L^{2}\left(Q_{0}\right)}=-\frac{1}{2} s(M / \nu) \iint_{Q_{0}} \alpha_{x t}|\psi|^{2} d x d t \geq-C s|M|\left(T_{0}^{3} / \nu\right) \iint_{Q_{0}} \alpha^{5}|\psi|^{2} d x d t$.

Furthermore,

$\left(\left(L_{1} \psi\right)_{4},\left(L_{2} \psi\right)_{4}\right)_{L^{2}\left(Q_{0}\right)}=3 s(M / \nu) \iint_{Q_{0}} \alpha_{x x}\left|\psi_{x}\right|^{2} d x d t \geq-C s|M|\left(T_{0}^{2} / \nu\right) \iint_{Q_{0}} \alpha^{3}\left|\psi_{x}\right|^{2} d x d t$.

Finally,

$$
\left(\left(L_{1} \psi\right)_{4},\left(L_{2} \psi\right)_{5}\right)_{L^{2}\left(Q_{0}\right)}=-\frac{1}{2} s\left(M^{2} / \nu^{2}\right) \iint_{Q_{0}} \alpha_{x x}|\psi|^{2} d x d t \geq-C s\left(T_{0}^{4} M^{2} / \nu^{2}\right) \iint_{Q_{0}} \alpha^{5}|\psi|^{2} d x d t .
$$

All these computations for $\left(L_{1} \psi\right)_{4}((144)-(148))$ show that

$$
\begin{aligned}
\left(\left(L_{1} \psi\right)_{4}, L_{2} \psi\right)_{L^{2}\left(Q_{0}\right)} & \geq-C|M|\left(s T_{0}^{2} / \nu\right)\left(s^{2}+T_{0}+T_{0}^{2}|M| / \nu\right) \iint_{Q_{0}} \alpha^{5}|\psi|^{2} d x d t \\
& -C s T_{0}^{2}|M| / \nu \iint_{Q_{0}} \alpha^{3}\left|\psi_{x}\right|^{2} d x d t-C s T_{0}^{2}|M| / \nu \int_{0}^{T_{0}} \alpha_{\mid x=1}^{3}\left|\psi_{x \mid x=1}\right|^{2} d t
\end{aligned}
$$

Let us now gather all the product $\left(L_{1} \psi, L_{2} \psi\right)_{L^{2}\left(Q_{0}\right)}$ coming from (131), (137), (143) and (149).

- As long as the zero order terms are concerned, we will get profit of the term obtained in (138) (or (143)):

$$
s^{5} \iint_{Q_{0}} \alpha^{5}|\psi|^{2} d x d t
$$

All terms concerning $|\psi|^{2}$ can be bounded by

$$
\left[s\left(T_{0}^{2}+\left(T_{0}^{4} M^{2} / \nu^{2}\right)\right)+s^{3}\left(T_{0}+T_{0}^{2}+\left(T_{0}^{2}|M| / \nu\right)\right)\right] \iint_{Q_{0}} \alpha^{5}|\psi|^{2} d x d t
$$


(see (131), (137), (143) and (149)). Then, taking $s \geq C\left(T_{0}+T_{0}^{1 / 2}+\left(T_{0}|M|^{1 / 2} / \nu^{1 / 2}\right)\right.$ ), all these integrals can be absorbed by (150).

- On the other hand, from (131) we have that the term involving $\left|\psi_{x x}\right|^{2}$ in $Q_{0}$ is

$$
-\frac{7}{2} s \iint_{Q_{0}} \alpha_{x x}\left|\psi_{x x}\right|^{2} d x d t \geq C s \iint_{Q_{0}} \alpha\left|\psi_{x x}\right|^{2} d x d t .
$$

The presence of (150) and (151) provides that the term

$$
C s^{3} \iint_{Q_{0}} \alpha^{3}\left|\psi_{x}\right|^{2} d x d t
$$

is also produced from $\left(L_{1} \psi, L_{2} \psi\right)_{L^{2}\left(Q_{0}\right)}$. This justifies that the terms on $\left|\psi_{x}\right|^{2}$ bounded by

$$
s T_{0}\left(1+\left(T_{0}|M| / \nu\right)\right) \iint_{Q_{0}} \alpha^{3}\left|\psi_{x}\right|^{2} d x d t
$$

(see (131), (137) and (149)) are absorbed as long as we take $s \geq C\left(T_{0}^{1 / 2}+\left(T_{0}|M|^{1 / 2} / \nu^{1 / 2}\right)\right.$ ).

- Concerning the traces, from (131) and (143) we have the terms

$$
4 s^{3} \int_{0}^{T_{0}} \alpha_{x \mid x=1}^{3}\left|\psi_{x \mid x=1}\right|^{2} d t
$$

and

$$
s \int_{0}^{T_{0}} \alpha_{x \mid x=1}\left|\psi_{x x \mid x=1}\right|^{2} d t
$$

The first one serves to eliminate

$$
-C s\left(T_{0}+T_{0}^{2}+T_{0}^{2}|M| / \nu\right) \int_{0}^{T_{0}} \alpha_{\mid x=1}^{3}\left|\psi_{x \mid x=1}\right|^{2} d t
$$

(see (131) and (149)) by taking $s \geq C\left(T_{0}+T_{0}^{1 / 2}+T_{0}|M|^{1 / 2} / \nu^{1 / 2}\right)$.

With all this, we get

$$
\begin{aligned}
& s \iint_{Q_{0}} \alpha\left(\left|\psi_{x x}\right|^{2}+s^{2} \alpha^{2}\left|\psi_{x}\right|^{2}+s^{4} \alpha^{4}|\psi|^{2}\right) d x d t+s \int_{0}^{T_{0}} \alpha_{\mid x=1}\left(\left|\psi_{x x \mid x=1}\right|^{2}+s^{2} \alpha_{\mid x=1}^{2}\left|\psi_{x \mid x=1}\right|^{2}\right) d t \\
& \leq C\left(s \int_{0}^{T_{0}} \alpha_{\mid x=0}\left|\psi_{x x \mid x=0}\right|^{2} d t+\left\|L_{3} \psi\right\|_{L^{2}\left(Q_{0}\right)}^{2}\right)
\end{aligned}
$$

for a choose of $s$ like $s \geq C\left(T_{0}+T_{0}^{1 / 2}+\left(T_{0}|M|^{1 / 2} / \nu^{1 / 2}\right)\right)$. Now, from the expression of $L_{3} \psi$ (see (124)) and (125), we see that

$$
\left\|L_{3} \psi\right\|_{L^{2}\left(Q_{0}\right)}^{2} \leq C\left(s^{2} T_{0}^{3}+s^{4} T_{0}\right) \iint_{Q_{0}} \alpha^{5}|\psi|^{2} d x d t
$$

which can also be absorbed by (150) with $s \geq C T_{0}$.

Finally, we come back to $\varphi$ by using the definition of $\psi=e^{-s \alpha} \varphi$ and the properties on the weight function $\alpha$ given in (56). As a consequence, we deduce estimate (59).

\subsection{Proof of Proposition 5}

Let us redefine the expressions of $L_{1} \psi, L_{2} \psi$ and $L_{3} \psi$ given in (122), (123) and (124):

$$
\begin{gathered}
L_{1} \psi=-\psi_{x x x}-\psi_{t}-3 s^{2} \alpha_{x}^{2} \psi_{x}, \\
L_{2} \psi=-s^{3} \alpha_{x}^{3} \psi-3 s \alpha_{x} \psi_{x x}-s \alpha_{t} \psi-3 s \alpha_{x x} \psi_{x}
\end{gathered}
$$


and

$$
L_{3} \psi=s \alpha_{x x x} \psi+3 s^{2} \alpha_{x} \alpha_{x x} \psi+(M / \nu) \psi_{x}+s(M / \nu) \alpha_{x} \psi .
$$

The double products of $L_{1} \psi$ and $L_{2} \psi$ are exactly the same as in the previous paragraph. This leads to the following estimate (similarly to (153)):

$$
\begin{aligned}
& s \iint_{Q_{0}} \alpha\left(\left|\psi_{x x}\right|^{2}+s^{2} \alpha^{2}\left|\psi_{x}\right|^{2}+s^{4} \alpha^{4}|\psi|^{2}\right) d x d t \\
& \leq C\left(s \int_{0}^{T_{0}} \alpha_{\mid x=0}\left|\psi_{x x \mid x=0}\right|^{2} d t+\iint_{Q_{0}}\left(|M|^{2} / \nu^{2}\right)\left(\left|\psi_{x}\right|^{2}+s^{2} \alpha^{2}|\psi|^{2}\right) d x d t\right),
\end{aligned}
$$

for $s \geq C\left(T_{0}, \nu\right)$.

Therefore, we only need to estimate the last two terms in the right hand side of (154) as long as $M \in L^{\infty}\left(0, T ; L^{2}(0,1)\right)$. Let us estimate, for instance, the first term:

$$
\iint_{Q_{0}}|M|^{2}\left|\psi_{x}\right|^{2} d x d t \leq \int_{0}^{T}\|M\|_{L^{2}(0,1)}^{2}\left\|\psi_{x}\right\|_{\infty}^{2} d t \leq\|M\|_{L^{\infty}\left(0, T ; L^{2}(0,1)\right)}^{2} \int_{0}^{T}\left\|\psi_{x}(t)\right\|_{L^{\infty}(0,1)}^{2} d t .
$$

Now, we take into account that there exists a positive constant $C$ such that

$$
\left\|\psi_{x}(t)\right\|_{L^{\infty}(0,1)} \leq C\left\|\psi_{x x}(t)\right\|_{L^{2}(0,1)} \quad \text { a. e. } t \in(0, T)
$$

and that $\alpha \geq 2 / T$. Consequently, we obtain

$$
\|M\|_{L^{\infty}\left(0, T ; L^{2}(0,1)\right)}^{2} \int_{0}^{T}\left\|\psi_{x}(t)\right\|_{L^{\infty}(0,1)}^{2} d t \leq C\|M\|_{L^{\infty}\left(0, T ; L^{2}(0,1)\right)}^{2} \iint_{Q_{0}} \alpha\left|\psi_{x x}\right|^{2} d x d t .
$$

Analogously, one can obtain the following estimate for the last integral in the right hand side of (154):

$$
s^{2} \iint_{Q_{0}}|M|^{2} \alpha^{2}|\psi|^{2} d x d t \leq C\|M\|_{L^{\infty}\left(0, T ; L^{2}(0,1)\right)}^{2} \iint_{Q_{0}} \alpha^{3}\left|\psi_{x}\right|^{2} d x d t .
$$

Then, taking $s \geq C\left(T_{0}, \nu,\|M\|_{L^{\infty}\left(L^{2}\right)}\right)$, we readily deduce (76) from (154).

Acknowledgements. Both authors are supported by Grant JCJC06_137283 of the Agence Nationale de la Recherche. The authors would like to express their gratitude to Lionel Rosier for useful discussions, and to the anonymous referee for useful comments on a preliminary version of this paper.

\section{References}

[1] Bergh J., Löfström J., Interpolation spaces. An introduction. Grundlehren der Mathematischen Wissenschaften, No. 223. Springer-Verlag, Berlin-New York, 1976.

[2] Bona J., Sun S. M., Zhang B.-Y., A nonhomogeneous boundary-value problem for the Korteweg-de Vries equation posed on a finite domain, Comm. Partial Differential Equations 28 (2003), no. 7-8, pp. 1391-1436.

[3] Cerpa E., Exact controllability of a nonlinear Korteweg-de Vries equation on a critical spatial domain, SIAM J. Control Optim. 46 (2007), pp. 877-899.

[4] Cerpa E., Crépeau E., Boundary controlability for the non linear Korteweg-de Vries equation on any critical domain, preprint 2007, to appear in Ann. Inst. H. Poincaré Anal. Non Linéaire.

[5] Colliander J. E. and Kenig C. E., The generalized Korteweg-de Vries equation on the half line, Comm. Partial Diff. Eq. 27 (2002), no. 11-12, pp. 2187-2266.

[6] Coron J.-M., Crépeau E., Exact boundary controllability of a nonlinear KdV equation with critical lengths, J. Eur. Math. Soc, 6, 2004, pp. 367-398. 
[7] Coron J.-M., Guerrero S., Singular optimal control: A linear 1-D parabolic-hyperbolic example, Asymp. Anal. 44 3,4 (2005), pp. 237-257.

[8] Faminskii A. V., On Two Initial Boundary Value Problems for the Generalized KdV Equation, Nonlinear Boundary Problems 14 (2004), pp. 58-71.

[9] Fursikov A., Imanuvilov O. Yu., Controllability of Evolution Equations, Lecture Notes \#34, Seoul National University, Korea, 1996.

[10] Glass O., Guerrero S., On the uniform controllability of the Burgers equation, SIAM J. Control Optim. 46 (2007), no. 4, pp. 1211-1238.

[11] Guerrero S., Lebeau G., Singular optimal control for a transport-diffusion equation, Comm. Partial Differential Equations 32 (2007), no. 12, pp. 1813-1836.

[12] Holmer J., The initial-boundary value problem for the Korteweg-de Vries equation, Comm. in Partial Differential Equations, 31 (2006), no. 7-9, pp. 1151-1190.

[13] Hörmander L., The analysis of linear partial differential operators. I. Distribution theory and Fourier analysis. Grundlehren der Mathematischen Wissenschaften 256. Springer-Verlag, Berlin, 1983.

[14] Lions J.-L., Exact controllability, stabilizability and perturbations for distributed systems, SIAM Review, 30 (1988), pp. 1-68.

[15] Rosier L., Exact boundary controllability for the Korteweg-de Vries equation on a bounded domain. ESAIM Control Optim. Calc. Var. 2 (1997), pp. 33-55.

[16] Rosier L., Lionel Exact boundary controllability for the linear Korteweg-de Vries equation on the half-line. SIAM J. Control Optim. 39 (2000), no. 2, pp. 331-351.

[17] Rosier L., Control of the surface of a fluid by a wavemaker. ESAIM Control Optim. Calc. Var. 10 (2004), no. 3, pp. 346-380.

[18] Russell D. L., Zhang B. Y., Controllability and stabilizability of the third-order linear dispersion equation on a periodic domain. SIAM J. Control Optim. 31 (1993), no. 3, pp. 659-676.

[19] Russell D. L., Zhang B. Y., Exact controllability and stabilizability of the Korteweg-de Vries equation. Trans. Amer. Math. Soc. 348 (1996), no. 9, 3643-3672.

[20] Smart D. R., Fixed point theorems, Cambridge Tracts in Mathematics, No. 66. Cambridge University Press, London-New York, 1974. 